\title{
Muscle-derived stem/progenitor cell dysfunction limits healthspan and lifespan in a murine progeria model
}

\author{
Mitra Lavasani 1,2, Andria R. Robinson ${ }^{3,4}$, Aiping Lu, ${ }^{1,2}$, Minjung Song 1,2, Joseph M. Feduska', Bahar Ahani ${ }^{1}$, \\ Jeremy S. Tilstra ${ }^{5}$, Chelsea H. Feldman ${ }^{3}$, Paul D. Robbins $2,3,5$, Laura J. Niedernhofer ${ }^{3,5}$ \& Johnny Huard ${ }^{1,2,5}$
}

With ageing, there is a loss of adult stem cell function. However, there is no direct evidence that this has a causal role in ageing-related decline. We tested this using muscle-derived stem/ progenitor cells (MDSPCs) in a murine progeria model. Here we show that MDSPCs from old and progeroid mice are defective in proliferation and multilineage differentiation. Intraperitoneal administration of MDSPCs, isolated from young wild-type mice, to progeroid mice confer significant lifespan and healthspan extension. The transplanted MDSPCs improve degenerative changes and vascularization in tissues where donor cells are not detected, suggesting that their therapeutic effect may be mediated by secreted factor(s). Indeed, young wild-type-MDSPCs rescue proliferation and differentiation defects of aged MDSPCs when co-cultured. These results establish that adult stem/progenitor cell dysfunction contributes to ageing-related degeneration and suggests a therapeutic potential of post-natal stem cells to extend health.

\footnotetext{
${ }^{1}$ Stem Cell Research Center, 206 Bridgeside Point II, 450 Technology Drive, Pittsburgh, Pennsylvania 15219, USA. ${ }^{2}$ Department of Orthopaedic Surgery, University of Pittsburgh School of Medicine, 3471 Fifth Avenue, Pittsburgh, Pennsylvania 15213, USA. ${ }^{3}$ University of Pittsburgh Cancer Institute, 5117 Centre Avenue, Hillman Cancer Center 2.6, Pittsburgh, Pennsylvania 15213, USA. ${ }^{4}$ Department of Human Genetics, University of Pittsburgh Graduate School of Public Health, 130 DeSoto Street, Pittsburgh, Pennsylvania 15261, USA. ${ }^{5}$ Department of Microbiology and Molecular Genetics, University of Pittsburgh School of Medicine, 523 Bridgeside Point II, 450 Technology Drive, Pittsburgh, Pennsylvania 15219, USA. Correspondence and requests for materials should be addressed to L.J.N. (email: niedlx@upmc.edu) or to J.H. (email: jhuard@pitt.edu).
} 
A universal characteristic of ageing is the loss of regenerative capacity leading to an impaired ability to respond to stress and, therefore, increased morbidity and mortality ${ }^{1}$. This has led to the hypothesis that ageing is caused, in part, by the loss of functional adult stem cells necessary for tissue regeneration ${ }^{2,3}$. Indeed, mice greater than two years of age have a significant reduction in the number and proliferative capacity of neural stem cells ${ }^{4,5}$ and male germline stem cells ${ }^{6,7}$. In contrast, with ageing, haematopoietic stem cell (HSC) numbers are preserved but their function is lost ${ }^{8,9}$. Muscle satellite cells also lose stem cell properties with ageing, but their abundance in old age remains controversial ${ }^{10-13}$. Ageingrelated changes in bone-marrow-derived mesenchymal stem cells (MSCs) include loss of proliferation and differentiation potential, increase in senescence and loss of capacity to form bone in vivo ${ }^{14,15}$. Similarly, MSCs derived from the bone marrow of patients with Hutchinson-Gilford progeria syndrome, a disease of dramatically accelerated ageing, are defective in their ability to differentiate ${ }^{16}$. Adipose-derived multipotent cells show an age-dependent loss of self-renewal capacity and an increased propensity for adipogene$\operatorname{sis}^{17}$. Despite this extensive evidence for adult stem cell decline with ageing, it is not known whether old stem cells contribute to ageingrelated degenerative changes or are merely a biomarker of it.

Given that ageing-related degenerative changes are universal in the musculoskeletal system ${ }^{18-20}$, we investigated the impact of ageing on murine muscle-derived stem/progenitor cells (MDSPCs). MDSPCs are multipotent cells isolated from postnatal skeletal muscle through an established preplate technique ${ }^{21,22}$. They have a capacity for long-term proliferation, are resistant to oxidative and inflammatory stress, show multilineage differentiation and selfrenew, induce neovascularization, and stimulate regeneration of bone, skeletal and cardiac muscles ${ }^{21,23-25}$. These cells likely reside in the perivascular niche ${ }^{26}$. These characteristics raise the possibility that the loss of MDSPCs or related perivascular progenitor cells could contribute to sarcopenia, osteoporosis ${ }^{27,28}$ and other degenerative diseases associated with ageing.

Here we examine MDSPC function in natural ageing and a mouse model of a human progeria. XFE progeroid syndrome is caused by mutations in XPF (xeroderma pigmentosum complementation group F) and characterized by dramatically accelerated ageing of the epidermal, haematopoietic, endocrine, hepatobiliary, renal, nervous, musculoskeletal and cardiovascular systems ${ }^{29} . X P F$ encodes one subunit of an endonuclease that together with its essential binding partner ERCC1 (excision repair cross complementation group 1) participates in numerous DNA repair mechanisms ${ }^{30}$. XFE progeroid syndrome was modelled in the mouse by mutation of the $m E r c c 1$ locus to titrate expression of ERCC1-XPF ${ }^{31,32}$. Ercc1 ${ }^{-1-}$ mice with undetectable levels of ERCC1-XPF have a severely reduced lifespan of 1 month $(\mathrm{mth})^{29,30,32}$ while $\operatorname{Ercc1}^{-/ \Delta}$ mice, expressing $10 \%$ of the normal level of ERCC1-XPF have a lifespan of $7 \mathrm{mths}$ (refs 30,32 ). Both strains faithfully mimic the systemic accelerated ageing of XFE progeroid syndrome and demonstrate a highly significant correlation with naturally aged mice at the level of histopathology, pathophysiology and genome-wide transcriptional reprogramming $30,32,33,34$. Our results indicate that MDSPC function is adversely affected with accelerated and natural ageing. This dysfunction directly contributes to ageing-related degenerative changes since transplantation of functional MDSPCs was sufficient to extend healthspan and lifespan of progeroid mice. Furthermore, we provide evidence that the therapeutic effect of MDSPCs may be mediated by secreted factors that act systemically.

\section{Results}

Loss of stemness properties with ageing. MDSPCs are slowly adhering cells isolated from skeletal muscle and enriched through a preplate technique ${ }^{21,22}$. Previously, we reported isolating these

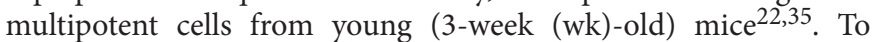

determine whether MDSPCs could also be isolated from adult and old mice, we followed the same preplate technique, and the resulting cells were analysed for expression of stem/progenitor cell markers (CD34, Sca-1). Cells isolated from skeletal muscle of young (3-wks), adult (19-wks), and old wild-type (WT) (2-years (yrs)) mice through this technique expressed CD34 and Sca-1 (Fig. 1a), establishing that MDSPCs could be isolated from mice of all ages. Although expression of CD34 appeared mildly attenuated in the older cell populations, Sca-1, which is a more stable marker of MDSPCs ${ }^{36}$, was similarly expressed in all three populations. Furthermore, MDSPCs isolated from adult WT mice showed multilineage differentiation (Fig. 1b).

To determine whether there is a loss of stem cell function with ageing and progeria, MDSPCs were isolated from old WT mice (2yrs) as well as progeroid $E r c c 1^{-1-}\left(2-3\right.$-wks) and $\operatorname{Erccl}^{-/ \Delta}$ (5-mths) mice and their proliferation and differentiation kinetics measured in vitro. As controls, MDSPCs were isolated from age-matched WT littermates of the progeroid mice and $\mathrm{Xpa}^{-1-}$ (xeroderma pigmentosum complementation group A) mice (3-wks), which are defective in nucleotide excision repair of DNA like ERCC1-deficient mice, but do not age rapidly ${ }^{37}$. To measure proliferation of MDSPCs, an equivalent number of cells from each population were plated, and live-cell imaging $(\mathrm{LCI})^{38}$ was used to measure growth kinetics (Fig. 1c). Analysis of images captured from the different cell populations revealed that proliferation of MDSPCs isolated from the old WT, $E r c c 1^{-/-}$and $E r c c 1^{-/ \Delta}$ mice, but not $\mathrm{Xpa}^{-/-}$mice, was significantly reduced relative to MDSPCs isolated from young WT mice $\left({ }^{\star} P<0.05\right.$, Tukey's test). The average population-doubling time (PDT) was prolonged in MDSPCs isolated from old mice [18hours (h)] and progeroid mice (20-27h) compared with young WT mice (15 h) (Fig. 1c). In support of this, the division time (DT) of single cells tracked within the population, or analysed as isolated clones, was also prolonged for old WT-MDSPCs and ERCC1deficient MDSPCs compared with young WT-MDSPCs (Supplementary Table S1). These data demonstrate that the proliferation capacity of MDSPCs diminishes with age.

To determine whether the capacity for myogenic differentiation is affected in old and progeroid MDSPCs, the cells were cultured to confluence and switched to differentiation medium (low serum). The young WT- and $\mathrm{Xpa}^{-1-}$ MDSPCs fused to form multinucleated myotubes expressing fast myosin heavy chain (f-MyHC), a marker of terminal myogenic differentiation (Fig. 1d). In contrast, MDSPCs isolated from old WT and progeroid ERCC1-deficient mice formed significantly fewer and smaller myotubes indicating impaired differentiation (Fig. 1d; ${ }^{\star} P<0.001$; Kruskal-Wallis ANOVA on ranks). The differentiation defect was confirmed by measuring the expression of myogenic differentiation markers MyHC, desmin, and myogenin (Fig. 1e). Myogenic differentiation of individual MDSPC clones from old and progeroid mice was also significantly reduced, compared with young WT mice (Supplementary Fig. S1; ${ }^{\star} P<0.001$, Kruskal-Wallis ANOVA on ranks). Similarly, osteogenic and chondrogenic differentiation of progeroid and old MDSPCs was attenuated compared with cells isolated from young WT mice (Fig. 1b). In contrast, the capacity for adipogenic differentiation was retained in aged MDSPCs. To determine whether the myogenic differentiation defect translated in vivo, MDSPCs isolated from progeroid mice were injected intramuscularly (IM) into $m d x$ mice, a model of Duchenne's muscular dystrophy ${ }^{39}$. Indeed, cells derived from $\operatorname{Ercc1}^{-/ \Delta}$ mice had an impaired ability to regenerate muscle fibres in dystrophic muscle compared with young WT-MDSPCs (Fig. 1f,g; $P<0.001$, Mann-Whitney rank sum test). Cumulatively, these data provide evidence of a significant loss of stemness properties, including proliferation under clonogenic conditions, multipotential differentiation and tissue regeneration, in MDSPC populations isolated from old and progeroid mice.

To rule out the possibility that the stem cell dysfunction was an artefact caused by extensive passaging of MDSPCs ex vivo, we 


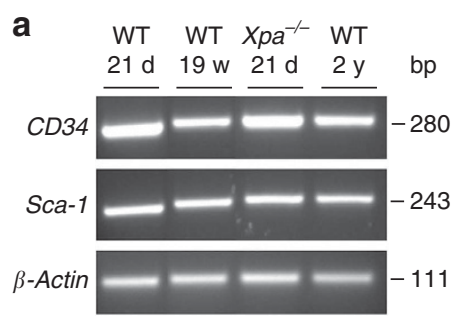

b

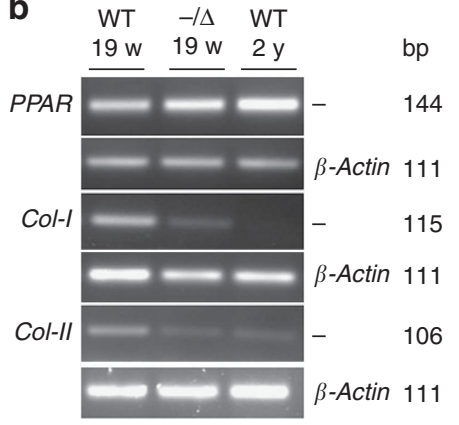

d
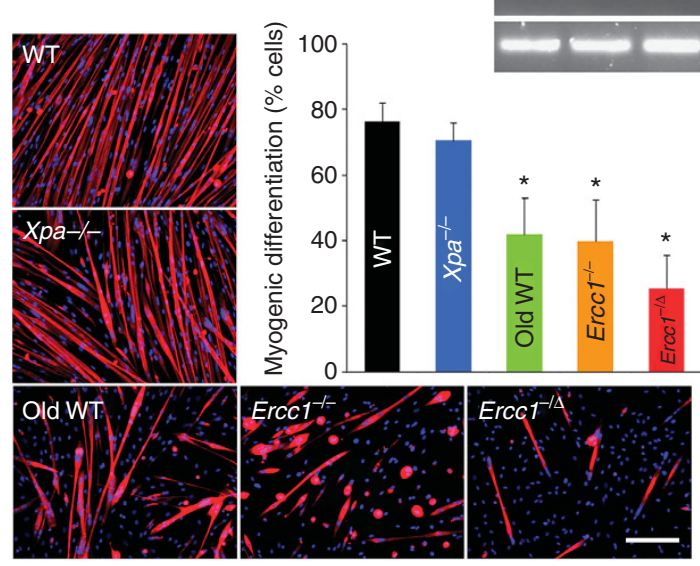

f

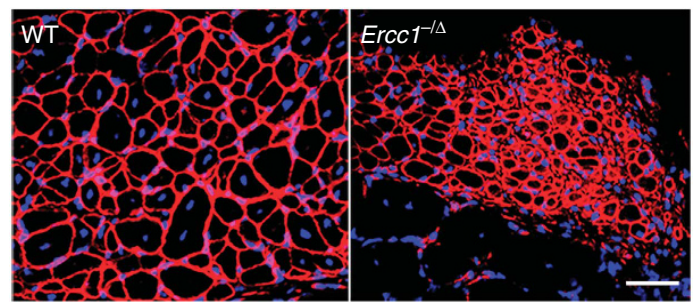

c
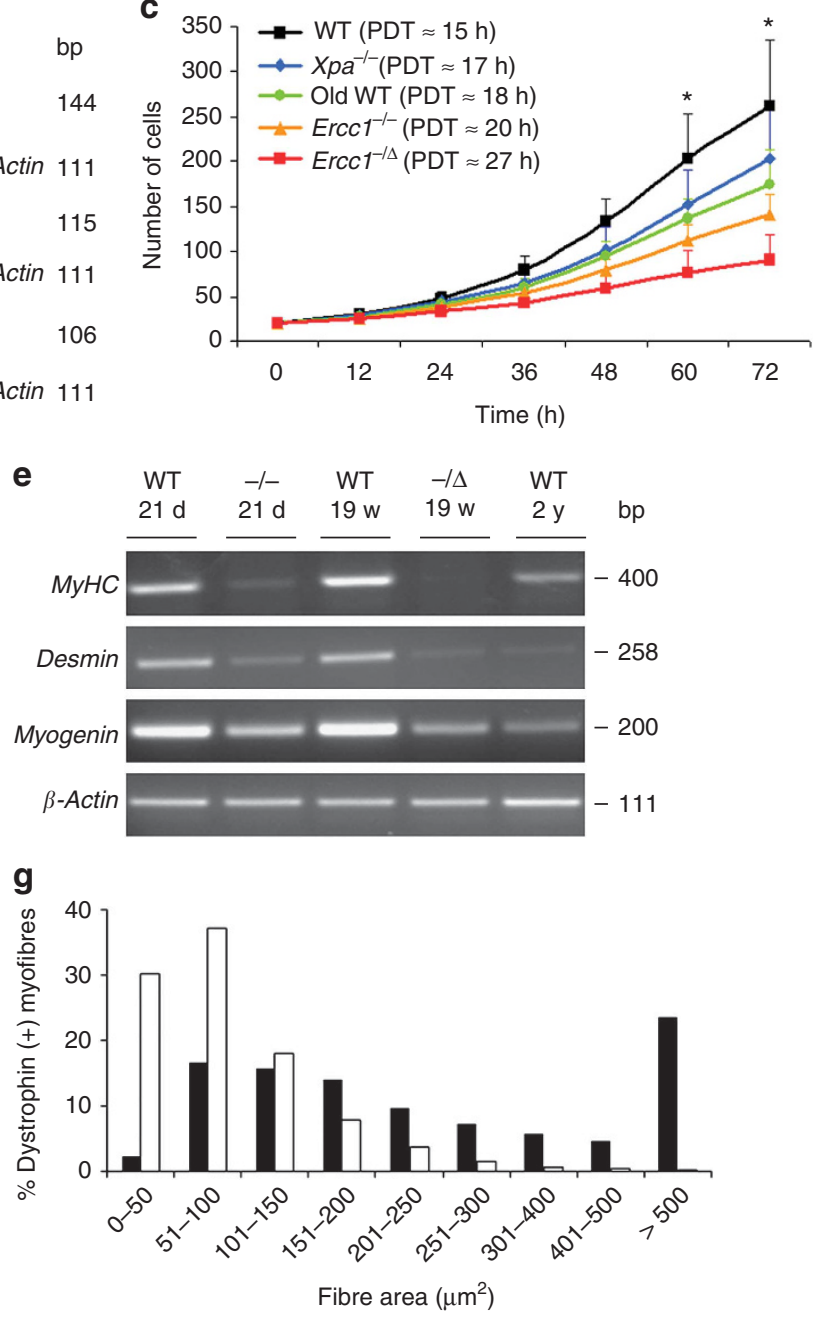

Figure 1 | Measuring MDSPC proliferation, differentiation, and regenerative potential. (a) rtPCR to measure stem/progenitor cell markers CD34 and Sca-1 in MDSPCs isolated from muscle of mice at various ages. Shown is a representative image from analysis of 3-4 independent MDSPC populations per genotype/age at passage 23-25. (b) rtPCR to measure differentiation markers. Peroxisome proliferator-activated receptor (PPAR) is an adipocyte marker; collagen 1 (Col-1) is an osteocyte marker; collagen-2 (Col-2) is a chondrocyte marker. $\beta$-Actin was the loading control. Shown is a representative image from analysis of three independent MDSPC populations of each genotype/age. (c) Proliferation of MDSPCs measured by live-cell imaging. Plotted are the average number of cells at each time point calculated from 3-4 populations per genotype \pm s.d. $\left({ }^{\star} P<0.05\right.$, Tukey's test). Average PDT was calculated from analysis of 40 images per time point. (d) Representative images of in vitro myogenic differentiation. Cells were immunostained for the terminal differentiation marker, $\mathrm{f}-\mathrm{MyHC}$ (red). Scale bar $=100 \mu \mathrm{m}$. Quantification of myogenic differentiation was calculated as the fraction of cells (DAPI, blue) expressing $\mathrm{f}-\mathrm{MyHC}$ (red) from 3-4 cell populations per genotype $\pm \mathrm{s}$.d; ${ }^{*} P<0.001$ relative to young WT-MDSPCs (black bar), Kruskal-Wallis ANOVA on ranks. (e) rtPCR to measure expression of the terminal differentiation markers, MyHC, desmin, and myogenin, after myogenic differentiation of MDSPCs isolated from mice of various genotypes/ages. Shown is a representative image from 3 MDSPC populations tested per group. (f) Representative image of gastrocnemius muscle sections from dystrophic mice 14 days post-injection of WT or Ercc1 ${ }^{-/ \Delta}$ MDSPCs to test myogenic differentiation in vivo. The sections were immunostained for dystrophin (red) to identify myofibres from donor cells. Scale bar $=100 \mu \mathrm{m}$. (g) Quantification of the donor myofibre area in dystrophic mice transplanted with WT-MDSPCs (black bars) or MDSPCs from Ercc1 ${ }^{-/ \Delta}$ mice (white bars). Plotted is the average number of myofibres of each size range calculated from $>2,000$ fibres analysed per $m d x / S C I D$ mouse $(n=8)$ treated with 2 independent MDSPC populations per genotype. ( $P<0.001$, for all distribution ranges except 101-150; Mann-Whitney rank sum test).

measured the number of cells with stem/progenitor surface markers and capacity for myogenic differentiation at the earliest possible time point following isolation from skeletal muscle. Preplate 1 and 2 (pp1-2; Fig. 2a), which constitute all cells directly isolated from skeletal muscle, were induced to differentiate within $24 \mathrm{~h}$ of isolation. f-MyHC positive myotube formation was impaired in cells isolated from the skeletal muscle of progeroid ERCC1-deficient mice compared with WT littermates (Fig. 2b). Similarly, expression of a second terminal myogenic differentiation marker, myogenin, was reduced in the ERCC1-deficient cells compared with WT controls (Fig. 2c). Twenty-four-hours post-isolation from skeletal muscle, those cells that remained nonadherent were stained for surface markers Sca-1,CD34 and CD 45 and analysed by fluorescenceactivated cell sorting (FACS) to quantify the mixed population of non-haematopoietic stem/progenitor cells in the muscle (Fig. 2d). Sca-1 is expressed on adult stem cells, a sub-population of myoblasts ${ }^{40}$, muscle-derived fibrogenic/adipogenic progenitors ${ }^{41}$, and a non-myogenic population of cells that emerge in muscle with ageing ${ }^{42}$.

In young WT mice, $\sim 20 \%$ of the cells were $\mathrm{Sca}-1^{+} / \mathrm{CD} 34^{+} /$ $\mathrm{CD} 45^{-}$. In contrast, $<10 \%$ of the cells isolated from old or progeroid skeletal muscle were $\mathrm{Sca}-1^{+} / \mathrm{CD} 34^{+} / \mathrm{CD} 45^{-}$. Averaging 


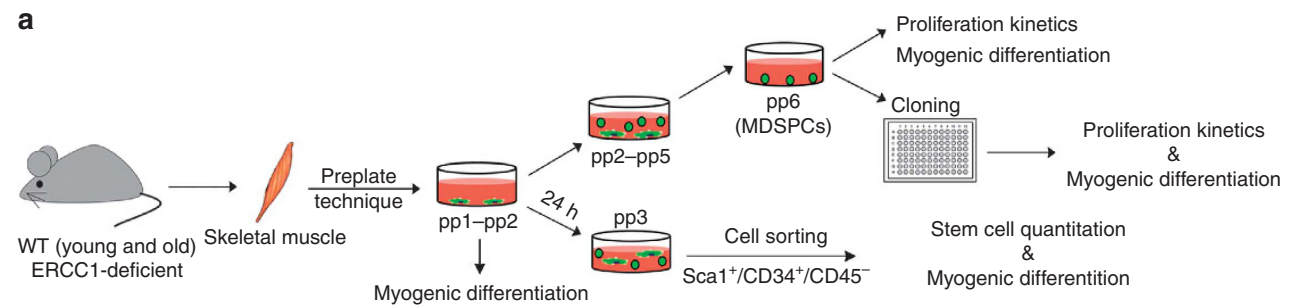

b WT $\mathrm{ErCC1}^{-1-}$ $\operatorname{Ercc1}^{-1 / \Delta}$
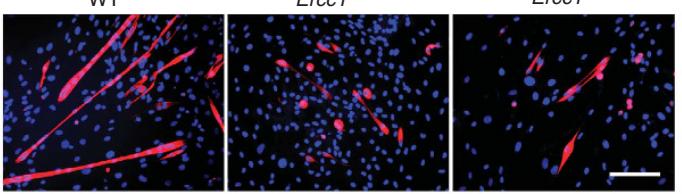

c
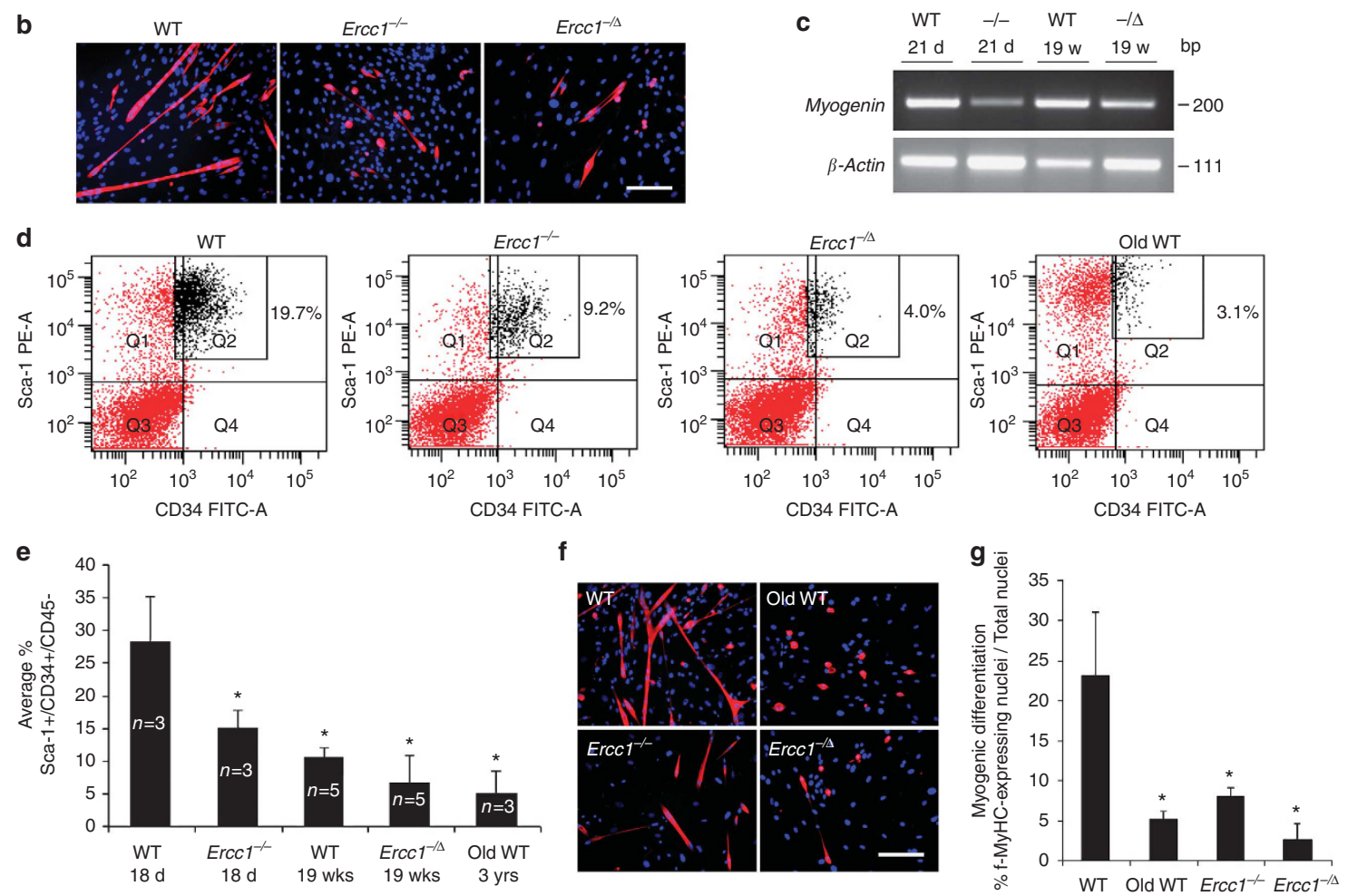

Figure 2 | Measuring the number of MDSPCs isolated from murine muscle and their myogenic potential. (a) A schematic diagram showing the early passage cells analysed in this figure and the method used to isolate various populations of MDSPCs (pp1-pp6). (b) Representative images of pp1-pp2 cells induced to undergo myogenic differentiation. Cells directly isolated from skeletal muscle of mice, using preplate technique (pp1-pp2), were incubated in fusion media to induce myogenic differentiation. Twenty-four hours post-isolation, adhering cells were trypsinized and plated at equal density and induced to undergo myogenic differentiation over 2-3 days. Cells from 2-3 independent populations of each genotype were immunostained for the terminal myogenic differentiation marker, $\mathrm{f}-\mathrm{MyHC}$ (red). Scale bar $=100 \mu \mathrm{m}$. (c) rtPCR to measure the expression of the myogenic differentiation marker, myogenin in pp1-pp2 cells, induced to undergo myogenic differentiation. (d) Quantification of Sca- $1^{+} / \mathrm{CD} 34^{+} / \mathrm{CD} 45^{-}$cells in young WT-, old WT-, and progeroid$\left(\mathrm{ErcC}^{-/-}, \mathrm{ErcC}^{-/ \Delta}\right)$ murine skeletal muscle. Twenty-four hours after isolation from muscle, the cells that did not adhere in preplate 1 and 2 (that is, pp3) were analysed for stem/progenitor cell markers by FACS. The number in the upper right quadrants indicates the percent of Sca- $1^{+} / \mathrm{CD} 34^{+} / \mathrm{CD} 45^{-}$cells isolated from 3-5 mice of each genotype/age. (e) Graph indicating the average fraction of Sca- $1^{+} / \mathrm{CD} 34^{+} / \mathrm{CD} 45^{-}$cells normalized to the weight of the cell pellet of pp3 cell populations. Error bars indicate \pm s.d. ${ }^{\star} P<0.05$, Tukey's test, relative to young WT cells. (f) Representative images of Sca- $1^{+} / \mathrm{CD} 34^{+} /$ $\mathrm{CD} 45^{-}$-sorted cells plated at equal density and induced to undergo myogenic differentiation. The cells were stained for the terminal myogenic marker, $\mathrm{f}-\mathrm{MyHC}$ (red). Scale bar $=100 \mu \mathrm{m}$. ( $\left(\right.$ ) Q Quantification of myogenic differentiation of Sca- $1^{+} / \mathrm{CD} 34^{+} / \mathrm{CD}_{4} 5^{-}$cells isolated from the skeletal muscle of mice of various genotypes/ages measured as the percent of cells (DAPI, blue) expressing f-MyHC (red). Error bars indicate \pm s.d. for cell populations isolated from 2-3 animals per genotype ${ }^{\star} P<0.001$, Kruskal-Wallis ANOVA on ranks relative to young WT cells.

data from 3-5 populations of each genotype revealed a significant depletion of this population of cells as animal age (Fig. $2 \mathrm{e} ;{ }^{\star} P<0.05$; Tukey's test). Next, we plated an equivalent number of these sorted cells to directly compare the myogenic differentiation capacity between $\mathrm{Sca}-1^{+} / \mathrm{CD} 34^{+} / \mathrm{CD} 45^{-}$cells from the different genotypes and ages of mice. Myogenic differentiation was significantly impaired in cells isolated from old or progeroid mice compared with young WT mice (Fig. 2f,g; ${ }^{\star} P<0.001$; Kruskal-Wallis ANOVA on ranks). These data demonstrate that there is a loss of MDSPC cell number as well as capacity for myogenic differentiation with ageing.
To determine whether there is a difference in the function of muscle stem cells between aged and young organisms in vivo, we examined muscle regeneration utilizing a well-established cardiotoxin injury-induced model (Supplementary Methods) ${ }^{43}$. The total area of muscle regeneration, as defined by centronucleated fibres, did not significantly differ between young WT, old WT, and progeroid mice (Supplementary Table S2). However, there was a dramatic difference in the average cross-sectional area of the regenerated myofibres in old and progeroid mice compared with young adult mice (Fig. 3a). More than $50 \%$ of the centronucleated, 
dystrophin-positive myofibres in old and progeroid mice had an area of $\leq 500 \mu \mathrm{m}^{2}$ (Fig. $3 \mathrm{~b}$ ). In contrast, $>60 \%$ of the myofibres in young WT mice had an area of $>1,000 \mu \mathrm{m}^{2}$, indicative of significantly more mature regenerated myofibres $(P<0.001$; Kruskal-Wallis ANOVA on ranks). In addition, Masson's Trichrome stain revealed increased fibrosis in muscle of old and progeroid mice in comparison to young WT mice following injury (Fig. $3 \mathrm{c}, \mathrm{d} ;{ }^{*} P<0.05$; Tukey's test). These data demonstrate that muscle regeneration is delayed and impaired in aged organisms. Collectively, the data in Figures 2 and 3 support the conclusion that aged organisms have a muscle stem/progenitor cell defect.

Treating with young WT-MDSPCs extends life and healthspan. To determine whether the loss of functional MDSPCs contributes to ageing-related degenerative changes, we asked whether transplantation of young WT-MDSPCs affects either the healthspan or lifespan of progeroid mice. Littermate pairs of $\mathrm{Erccl}^{-/-}$mice were injected
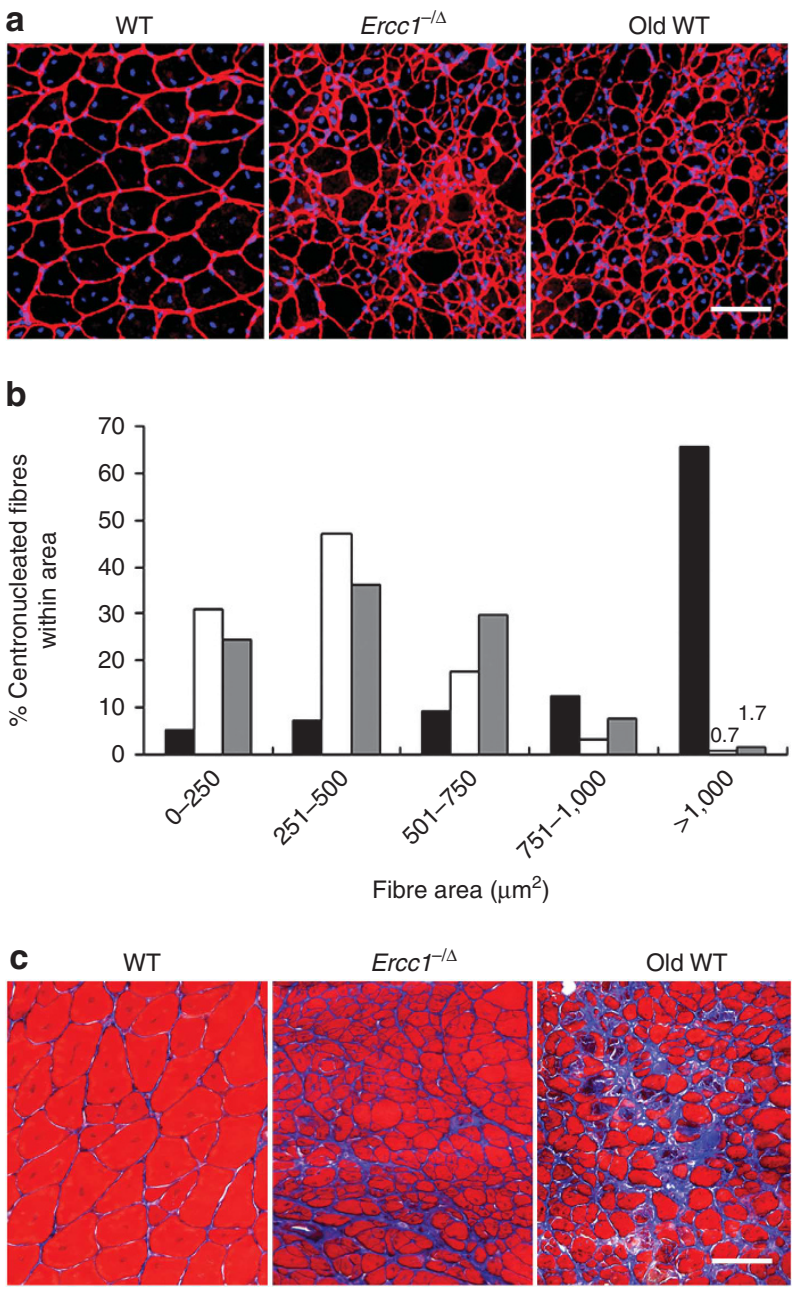

d

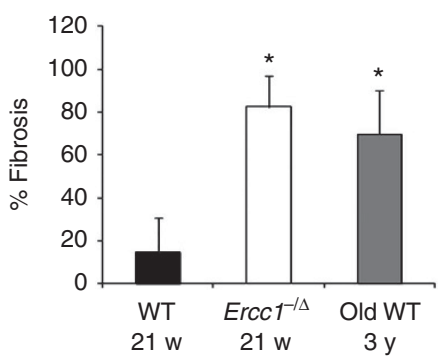

intraperitoneally (IP) with $2-4 \times 10^{5}$ young WT-, progeroid-, or old WT-MDSPCs per gram body weight or PBS at 17 days of age, and lifespan was measured (summarized in Supplementary Table S3). Ercc1 ${ }^{-1-}$ mice injected with young WT-MDSPCs showed pronounced weight gain and a significantly extended lifespan compared with sibling mutant animals injected with vehicle only (Fig. $4 \mathrm{a} ;{ }^{\star} P<0.05$, Dunn's test). In an identical experiment, early passage primary mouse embryonic fibroblasts (MEFs) did not affect the growth or lifespan of Ercc1 ${ }^{-/}$mice (Fig. 4a). Similarly, MDSPCs isolated from old WT or progeroid $\mathrm{Erccl}^{-/}$- mice did not have a significant impact on lifespan.

The young WT-MDSPCs were also injected IP into $\operatorname{Ercc1}^{-/ \Delta}$ mice at 6-7 wks of age and again 6 wks later. The age at onset of characteristic progeroid symptoms was measured biweekly, including dystonia, trembling, kyphosis, ataxia, muscle wasting, loss of vision, urinary incontinence and decreased spontaneous activity. The results were compared with littermate $\operatorname{Erccl}^{-/ \Delta}$ mice treated with vehicle only in a pairwise fashion. The investigators were blinded to the treatment arm. The ageing score, representing the fraction of symptoms that were delayed in mutant mice treated with MDSPCs compared with a mutant littermate treated with PBS, revealed a highly significant difference between treatment groups (Fig. 4b; ${ }^{* *} P<0.0008$; Student's $t$-test). These data provide evidence that loss of stem cell function has a direct causal role in ageingrelated degeneration.

Young WT-MDSPCs engraft after transplantation. To address the question of how MDSPCs could extend healthspan and lifespan, we asked whether the donor cells engrafted in host tissues. Young WT-MDSPCs, transduced with a retroviral vector containing LacZ with a nuclear localization sequence ( $n L a c Z)$, were injected IP into $\operatorname{Ercc1} 1^{-1-}$ mice $(n=8)$. Fourteen tissues were analysed (Supplementary Table S4) for the presence of donor cells. LacZ+ cells were detected in the parenchyma of the liver and kidney, as well as connective tissues associated with pancreas and spleen of all mice analysed (Fig. 5). Similar results were obtained in animals euthanized from 1 to 9 wks post-injection. LacZ + cells were also detected in lung, oesophagus, thymus, bladder/ureter, spinal cord, and bone marrow of at least one mouse (Fig. 5; Supplementary Fig. S2). Importantly, donor cells were not detected in the heart, brain, skeletal muscles, and lymph nodes of any mice. The extent of donor cell engraftment and expansion is limited, indicating that the beneficial effects of MDSPCs is not due to tissue reconstitution

Figure 3 | Measuring muscle regeneration after injury in old and progeroid mice. To measure muscle stem/progenitor cell function in vivo, cardiotoxin was injected directly into the gastrocnemius muscle of old WT (3-yr-old; $n=3)$, adult WT (18-21-wk-old; $n=5)$, and progeroid Ercc1-/ (21-wk-old; $n=2$ plus 8-wk-old; $n=3$ ) mice to induce muscle injury. Two wks later, tissues were collected and stained for dystrophin (red) and nuclei (DAPI). (a) Representative images of damaged muscle sections. Scale bar $=100 \mu \mathrm{m}$. (b) Quantification of the cross-sectional area of regenerating myofibres after muscle injury of adult WT mice (black bars), progeroid $\mathrm{ErcCl}^{-/ \Delta}$ littermates (white bars) and old WT mice (grey bars). The area of $>1,000$ myofibres was measured per group of mice. The distribution of fibre size is indicated on the $x$-axis, representing increasingly more mature fibres with increased size. The fraction of regenerating, centronucleated myofibres of each size range is plotted. $(P<0.001$, Kruskal-Wallis ANOVA on ranks at $0-250,251-500$, and $>1,000$ ). (c) Trichrome staining of sections from the same damaged muscles to reveal areas of fibrosis (blue). Shown are representative images from 1 of 2-3 mice of each genotype analysed. (d) Histogram indicating the area of fibrotic scarring following cardiotoxin injection. Plotted is the average fraction ( $n=2-3$ animals per genotype/age) of the area examined that is fibrotic in percent \pm s.d. ${ }^{\star} P<0.05$, Tukey's test, relative to young WT. Scale bar $=100 \mu \mathrm{m}$. 

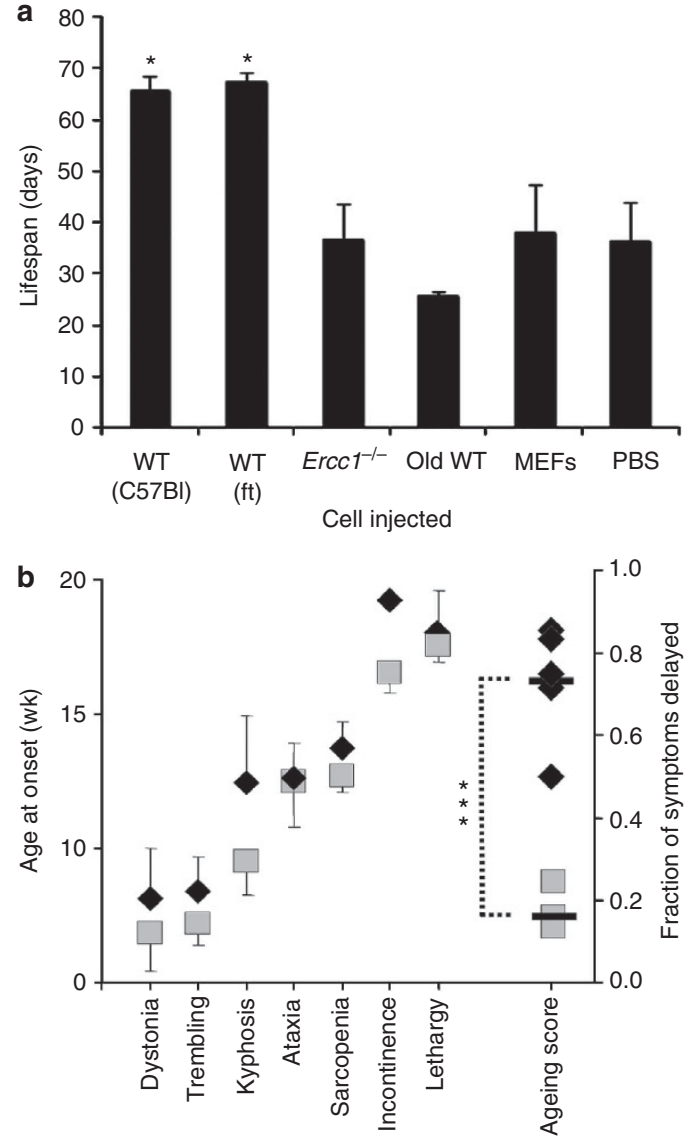

Figure 4 | Measuring the impact of MDSPC transplantation on the lifespan and healthspan of progeroid mice. (a) $2-4 \times 10^{5}$ MDSPCs per gram body weight were injected into the peritoneal cavity of 17-day-old $\mathrm{ErcCl}^{-/-}$mice and lifespan measured. The median lifespan of $\mathrm{Erccl}^{-/-}$ mice is 21 days; the maximum is 28 days $^{29}$. The lifespan of $\mathrm{Erccl}^{-/-}$mice injected with two independent MDSPC populations isolated from young WT mice were compared with $\mathrm{Ercc}^{-/-}$mice injected with vehicle only (PBS), an equivalent number of WT MEFs, or MDSPCs isolated from old WT or progeroid $\mathrm{Ercc}^{-/-}$mice. Reported is the average lifespan from 4-10 mice injected per treatment group. Error bars indicate s.e.m. ${ }^{\star} P<0.05$, Dunn's test comparing the WT groups with all other treatment groups. A summary of the cell lines injected and number of animals transplanted can be found in Supplementary Table S3. (b) $2-4 \times 10^{5}$ MDSPCs per gram body weight were injected into the peritoneal cavity of 7-wk-old Ercc1-/ mice and again at $13 \mathrm{wks}$ of age. The age at onset of the characteristic, spontaneous, ageing-related symptoms seen in $\mathrm{Erccl}^{-/ \Delta}$ mice was measured (MDSPC-treated mice, black diamonds) and compared with mice injected with vehicle only (PBS; grey squares). Reported is the average age at onset of each progeroid symptom in wks \pm s.d. ( $n=8$ mice per treatment group). The ageing score is calculated as the fraction of symptoms that were delayed in a single $\mathrm{ErcC}^{-/ \Delta}$ mice injected with young WT-MDSPCs compared with an age- and sex-matched mouse (usually a littermate) injected with PBS (Supplementary Methods). The black bars denote the average ageing score for each group ( ${ }^{\star \star \star} P<0.0008$, Student's $t$-test)

by donor cells as is seen with haematopoietic stem cells in bone marrow transplant.

Young WT-MDSPCs restore the function of aged MDSPCs. In a progeria model (Ercc1 $1^{-/}$mice), young WT-MDSPCs had a therapeutic effect. However, the duration of the experiment ( $<3 \mathrm{wks}$ ) and the limited extent of engraftment of IP-injected MDSPCs, as far as we could detect, made it unlikely that this positive effect was a result of substantial MDSPC engraftment, proliferation, differentiation and tissue regeneration. Thus, we next asked whether young WT-MDSPCs confer their therapeutic effects through a paracrine mechanism, requiring secreted factors. MDSPCs isolated from 14-day-old progeroid Ercc1 ${ }^{-/}$mice were co-cultured with the young WT-MDSPCs, using a transwell system (Fig. 6a). The proliferation of $\mathrm{Ercc1}^{-1-}$ MDSPCs was measured for $72 \mathrm{~h}$ while the cells were exposed to proliferation media from young WT-MDSPCs. Subsequently, the $\mathrm{Ercc1}^{-/-}$MDSPCs were switched to low-serum media to measure myogenic differentiation. The proliferation defect of $\mathrm{Ercc1}^{-1-}$ MDSPCs was significantly improved by coculture of the cells with young WT-MDSPCs (Fig. 6 b; ${ }^{\star} P<0.001$, Mann-Whitney rank sum test). In addition, myogenic differentiation of $\mathrm{Ercc1}^{-1-}$ MDSPCs was improved after co-culture with young WT-MDSPCs (Fig. 6c). Likewise, impaired differentiation of old WT-MDSPCs was rescued by conditioned media from young WT-MDSPCs. To quantify the improvement, MDSPCs from progeroid $\mathrm{Ercc1}^{-1-}$ or old WT mice were grown in the presence of conditioned media from young WT-MDSPCs, then collected for immunodetection of the terminal myogenic differentiation marker f-MyHC (Fig. 6d). f-MyHC expression was dramatically increased $(2.5-9 \times)$ if the cells were grown in the presence of media from young WT-MDSPCs compared with differentiation media alone. Thus young WT-MDSPCs were able to rescue, at least in part, the function (proliferation and differentiation) of MDSPCs isolated from progeroid and old mice via secreted factors.

MDSPCs promote neovascularization and tissue regeneration. To determine whether secreted factors were indeed important for the therapeutic effect of MDSPCs, we asked whether there was evidence of improved histopathology in tissues of treated ERCC1deficient mice in which transplanted MDSPCs were not detected, in particular, skeletal muscle and brain. We previously demonstrated that the regenerative potential of MDSPCs transplanted into skeletal muscle, heart and bone correlates with their ability to induce angiogenesis $21,24,25,35,44$. Thus, we asked whether IP injection of young WT-MDSPCs had an effect on the vasculature of the brain, as well as muscle fibre size. Muscle sections were stained for dystrophin to highlight muscle fibre perimeter and CD31 to identify endothelial cells within microvessels (Fig. 7a). For each image, the total number of muscle fibres and CD31 + cells were quantified, and the ratio (\# of CD31 + cells/\# of muscle fibres) determined. Muscles of 15-day-old $\mathrm{Ercc}^{-/-}$mice had significantly reduced numbers of CD31 + cells at the periphery of muscle fibres compared with WT littermates (Fig. 7b; 0.56 CD31 + cells per fibre versus 1.25 for WT mice; ${ }^{\star} P<0.001$, Student's $t$-test). The average muscle-fibre size was also significantly reduced in the $\operatorname{Erccl}^{-/-}$mice $\left(324 \mu \mathrm{m}^{2}\right)$ compared with WT littermates (Fig. $7 \mathrm{~b} ; 734 \mu \mathrm{m}^{2},{ }^{\star} P=0.002$, Mann-Whitney rank sum test).

IP injection of young WT-MDSPCs into $\mathrm{Erccl}^{-/-}$mice led to a significant increase in the number of CD31 + cells 9 wks postinjection compared with untreated $\mathrm{Erccl}^{-1-}$ mice that only live 3-4 wks (Fig. 7b; $1.05 \mathrm{CD} 31+$ vessels/fibre; ${ }^{\S} P<0.05$, Tukey’s test). Similarly, muscle-fibre size significantly increased (Fig. 7b; $1,580 \mu \mathrm{m}^{2}$ cross-sectional area; ${ }^{\circledR} P<0.05$, Dunn's test). It is impossible to directly compare the fibre size between $\operatorname{Erccl}^{-/-}$mice transplanted with young WT-MDSPCs to untreated mutant animals of the same age because the latter don't live to 9 wks of age (Fig. 4a). Nevertheless, muscle regeneration and growth was evident in $\operatorname{Ercc1}^{-/-}$mice treated with MDSPCs.

Progeroid $\mathrm{Erccl}^{-1-}$ mice show reduced vascularization in the brain compared with WT littermates at 3 wks of age (Fig. 7c; Supplementary Fig. S3). Although donor cells were not detected in the brain of $\operatorname{Ercc}^{-1-}$ mice, cell transplantation significantly improved neovascularization in the brain of these progeroid mice. In 3-wkold $E r c c 1^{-/-}$mice, the median area of cerebral cortex occupied by 
a

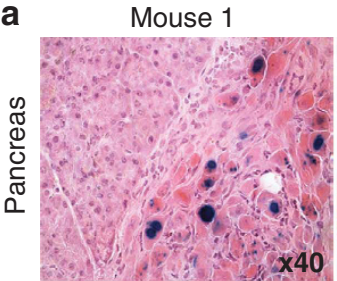

Mouse 2

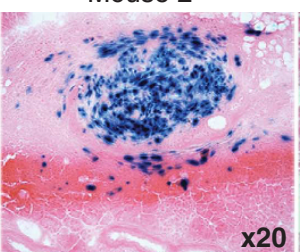

Mouse 3

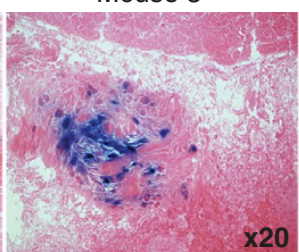

Mouse 4
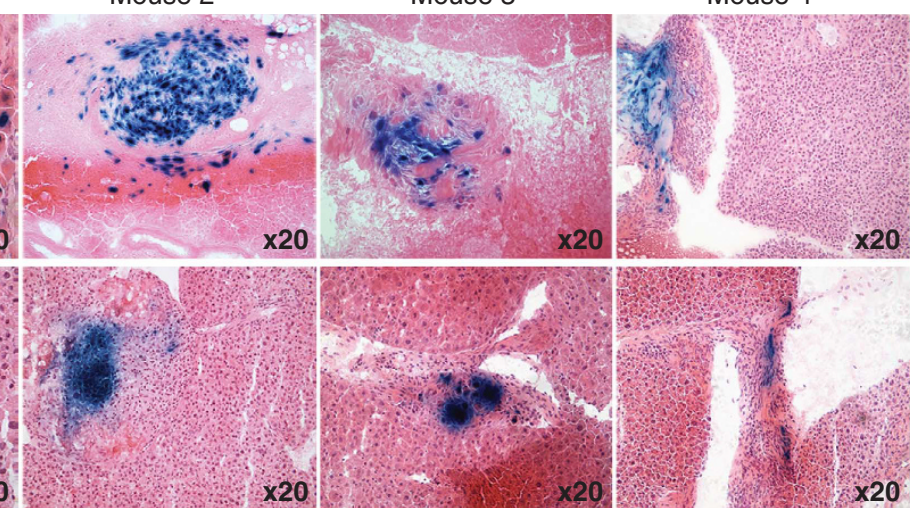

$x 20$
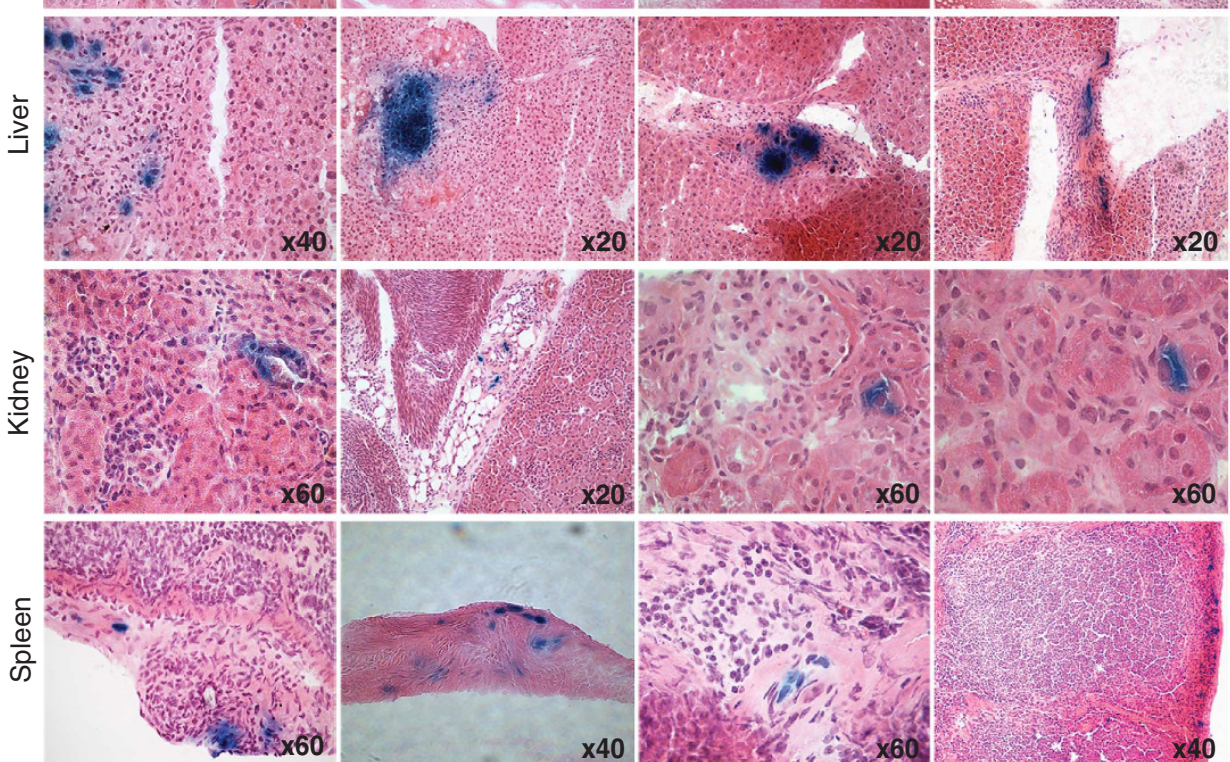

b
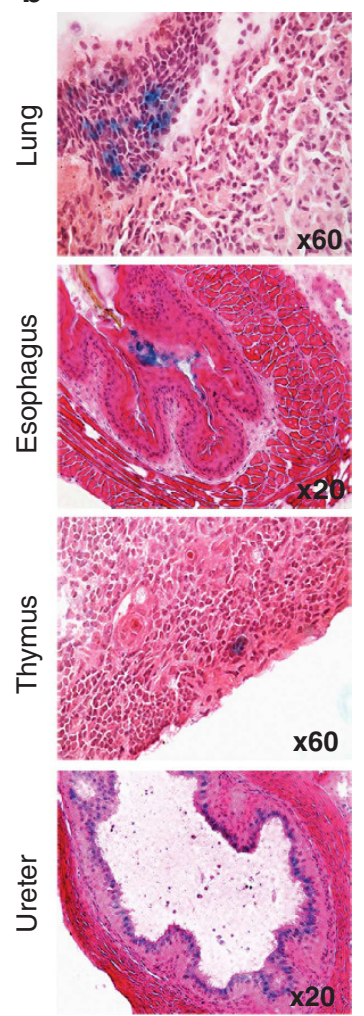

Figure 5 | Determination of the site for donor cell engraftment of young WT-MDSPCs. 2-4 $\times 10^{5}$ young WT-MDSPCs expressing nuclear LacZ per gram body weight were injected IP into $\mathrm{ErcC}^{-/-}$mice to determine the sites of engraftment. Mice were injected at 12 days of age and euthanized 8-9 days later $(n=4)$ or injected at 17-19 days of age and tissues collected at the end of their lifespan (4-9-wks of age; $n=4)$. Fourteen organs/tissues were isolated, sectioned and stained with X-gal to identify donor cells (Supplementary Table S4). (a) Donor cells (LacZ+ stained blue) were detected in the pancreas, liver, spleen, and kidney of all host animals. Shown are representative images from four mice illustrating the site and extent of engraftment of donor cells. (b) Donor cells were detected in the lung, esophagus, thymus and ureter of at least one mouse. Shown are images at multiple levels of magnification from tissue sections of one mouse per organ to illustrate the site and extent of engraftment. The level of magnification is indicated for each tissue section image.

vasculature was $1.2 \%$. In $E r c c 1^{-1-}$ mice injected with MDSPCs, vascularization increased approximately to the levels in 3-wk-old WT mice (2.2 versus $2.5 \%$, respectively) and the improvement was sustained. $\mathrm{Erccl}^{-1-}$ mice treated with MDSPCs that survived to 9 wks of age, had a vascular area indistinguishable from age-matched WT mice (3.2 versus $3.1 \%, P<0.05$, Dunn's test). These data demonstrate that there is improvement in the histopathology of several tissues in which donor cell engraftment was not detected, suggesting that MDSPCs exert their therapeutic effect through secreted factors.

Using IM injection of $n L a c Z$-labelled MDSPCs to localize donor-cell engraftment, we analysed host muscle for presence of $\mathrm{LacZ}+$ myofibres and endothelial cells (Fig. 8). Like IP, IM administration of young WT-MDSPCs into $\operatorname{Ercc1}^{-1-}$ mice (Fig. 8a, $n=3$ mice per group) led to a significant increase in the number of CD $31+$ cells (Fig. 8b; 1.06 CD $31+$ vessels per fibre; ${ }^{\S} P<0.05$, Tukey's test) compared with the non-injected $\operatorname{Erccl}^{-/-}$mice $(0.56$ $\mathrm{CD} 31+$ vessels/fibre). The muscle-fibre cross-sectional area was also significantly increased (Fig. 8b; 522 versus $324 \mu \mathrm{m}^{2},{ }^{\S} P<0.05$, Dunn's test). At the site of injection, numerous myofibres with central or peripheral $L a c Z+$ nuclei were identified, demonstrating that transplanted MDSPCs have the capacity to integrate into regenerated muscle fibres (Fig. 8c). In addition, analysis of sections of the gastrocnemius distant from the injection site revealed a shift in the distribution of myofibre size towards larger regenerating fibres compared with contralateral non-injected muscle (Fig. 8d;
$P<0.001$, Mann-Whitney rank sum test at 151-300 and 301-450). This suggests that IM injection of MDSPCs also affected host myofibres through a paracrine mechanism, requiring secreted factors. Sections were also stained with X-gal and for CD31 to determine whether donor cells co-localize with CD31+ cells (Fig. 8e). Background staining in $\mathrm{Erccl}^{-/-}$mice was extensive, compared with $m d x /$ SCID mice, likely due to interstitial fibrosis. In both cases, there was a lack of co-localization of LacZ and CD31 staining. These data are consistent with our previous findings ${ }^{25,45}$ and the conclusion that transplanted MDSPCs do not directly contribute to blood vessels and thus likely drive host angiogenesis through secreted factors.

\section{Discussion}

MDSPCs are stem/progenitor cells isolated from skeletal muscle, a tissue that universally declines in mass and function with ageing ${ }^{20}$. This cell population cannot be discriminated from less multipotent progenitors strictly based on surface markers, and thus are difficult to quantify. Nevertheless, MDSPCs were isolated from old WT mice and mice with accelerated ageing, using a well-established preplate technique ${ }^{21,22}$. In both cases, cell proliferation and multilineage differentiation is significantly impaired relative to MDSPCs isolated from young WT mice (Fig. 1). Furthermore, fewer cells expressing stem/progenitor cell markers are isolated from skeletal muscles of aged and progeroid mice (Fig. 2). Finally, muscle from aged and progeroid mice has significantly reduced regenerative capacity 
a

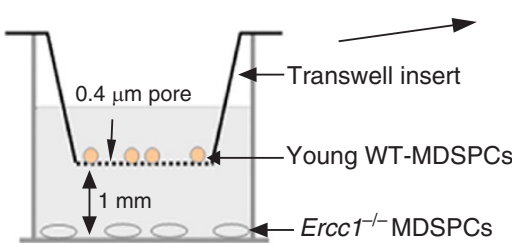

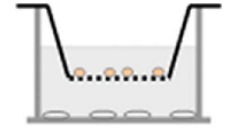

Proliferation ( $\mathrm{LCl}$ system for $72 \mathrm{~h}$ )

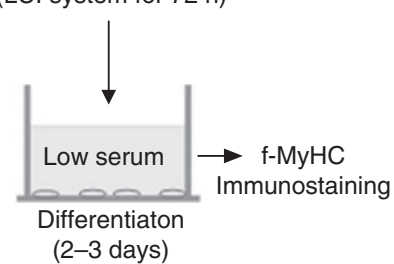

(2-3 days)

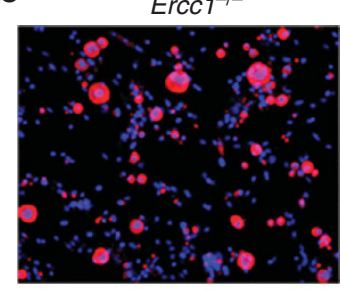

Old WT

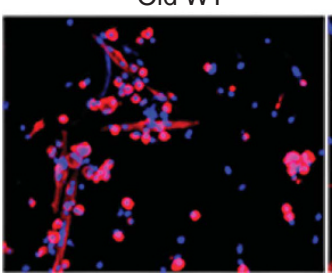

Ercc1 ${ }^{-1-}+$ Young WT

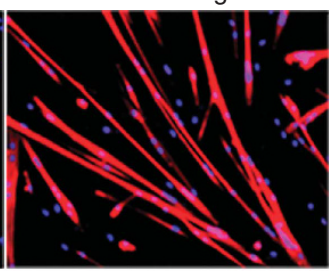

Old WT + Young WT

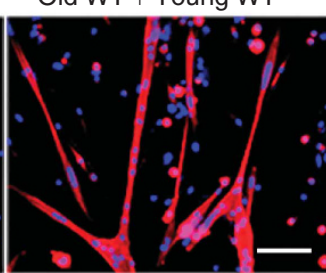

d

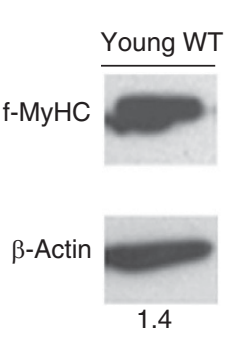

1.4 b

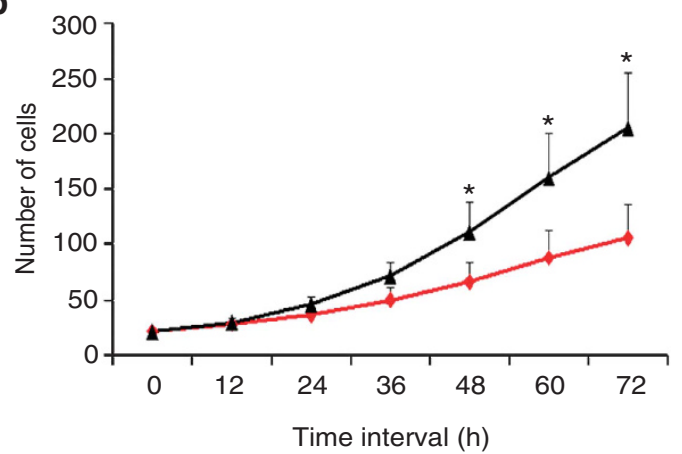

$\mathrm{Ercc1}^{-1-}$
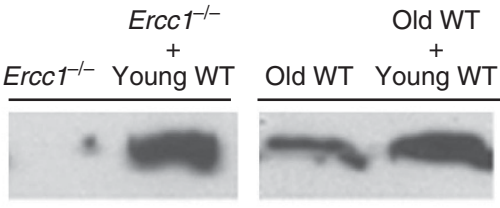

200 kDa
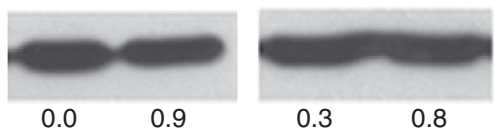

$42 \mathrm{kDa}$

Expression relative to $\beta$-Actin
Arbitrary

units

Figure 6 | Measurement of proliferation and myogenic differentiation of MDSPCs co-cultured with young WT-MDSPCs. (a) A schematic diagram of the co-culture system used to evaluate the effect of young functional MDSPCs on dysfunctional MDSPCs isolated from progeroid or old WT mice. Ercc $1^{-/-}$ MDSPCs were plated in the lower compartment of the transwell system in proliferation media. WT-MDSPCs were seeded onto the upper transwell membrane inserts, at the same density, and in the same media. These co-cultures were placed in the $\mathrm{LCl}$ system for $72 \mathrm{~h}$ to acquire time-lapsed images to measure proliferation of the MDSPCs. As a control, each plate contained wells of $\mathrm{rrcc}^{-1}{ }^{-}$MDSPCs without transwell membrane inserts.

(b) Plotted is the average cell number at each time point calculated from the analysis of three independent populations of Ercc $1^{-/-}$MDSPCs CO-cultured with young WT-MDSPCs (black line), or without (red line) \pm s.d. ${ }^{\star} P<0.001$, Mann-Whitney rank sum test. (c) The transwell inserts were removed after $72 \mathrm{~h}$ and the proliferation media was switched to differentiation media. After 2-3 days, myogenic differentiation of Ercc $1^{-/{ }^{-}}$and old WT-MDSPC was tested by immunostaining the cells for $\mathrm{f}-\mathrm{MyHC}$ (red). Shown are representative images. The nuclear counterstain is DAPI (blue). Scale bar $=100 \mu \mathrm{m}$. (d) Quantification of myogenic differentiation of old WT-MDSPCs after growth in media conditioned from young WT-MDSPCs. Young WT-MDSPCs were cultured for 2 days in proliferation media in collagen-coated flasks, then treated with differentiation media for 3 days. The supernatant from these cultures was collected for use as conditioned media. MDSPCs from 21-day-old progeroid Ercc1-/- mice and 2-yr-old WT mice were grown in the presence of this conditioned media or unconditioned differentiation media to determine the impact on myogenic differentiation as measured by immunoblot detection of $\mathrm{f}-\mathrm{MyHC}$. Densitometric quantification of $\mathrm{f}-\mathrm{MyHC}$ corrected for $\beta$-Actin is indicated below each lane.

following injury (Fig. 3; Supplementary Table S2), consistent with previous reports ${ }^{11}$. These data establish that the function of MDSPCs is compromised with ageing, similar to neuronal, mesenchymal, haematopoietic, satellite, and adipose stem cells ${ }^{4,15,17,46-48}$. The similarity of deficits in MDSPCs from old WT and progeroid mice extends the commonalities between accelerated ageing caused by a defect in DNA repair and natural ageing. Similar parallels were found between Hutchinson-Gilford progeria syndrome and ageing in the MSC compartment ${ }^{49}$.

Numerous studies provide evidence that the number and/or function of diverse adult stem cell populations decline with ageing 4,6,7,15,47,50,51. However, these are largely correlative studies that do not discriminate whether changes in the stem cell compartment have a causative role in ageing or are merely a consequence of ageing. Genetic studies revealed proteins and pathways essential for the maintenance of stem cell function. Mutation of these genes leads to foreshortened lifespan and early onset of some ageingrelated pathologies. For example, mice deficient in the polycomb protein BMI-1 have a short lifespan ${ }^{52}$, along with defects in self- renewal of HSCs, leading to adult stem cell depletion ${ }^{53}$. Similarly, mice deficient in HMGA2 show reduced stem cell numbers and function (self-renewal) throughout the central and peripheral nervous systems ${ }^{54}$. Finally, HSC function and regenerative capacity are significantly diminished in mice harbouring mutations in diverse DNA repair genes or telomerase (Lig4 ${ }^{\mathrm{Y} 288 \mathrm{C}}, \mathrm{Ku} 80^{-1-}, \mathrm{Xpd}^{\mathrm{TTD}}$, and $\left.m T R^{-1-}\right)$ indicating that genome and telomere maintenance are crucial for stem cell function ${ }^{55,56}$. However, there is no evidence that loss of stem cell function is directly responsible for decreased lifespan and ageing-related pathology in these models. Here we demonstrate that transplantation of functional WT stem/progenitor cells isolated from juvenile mice into hosts harbouring dysfunctional MDSPCs leads to a significant extension of healthspan and lifespan (Fig. 4). These results provide experimental evidence that the loss of adult stem cell function directly contributes to ageingrelated phenotypes.

Interestingly, the function of MDSPCs from aged mice could be restored if they were co-cultured with young WT-MDSPCs (Fig. 6). Similar results were seen in vivo using parabiosis ${ }^{47}$. These obser- 

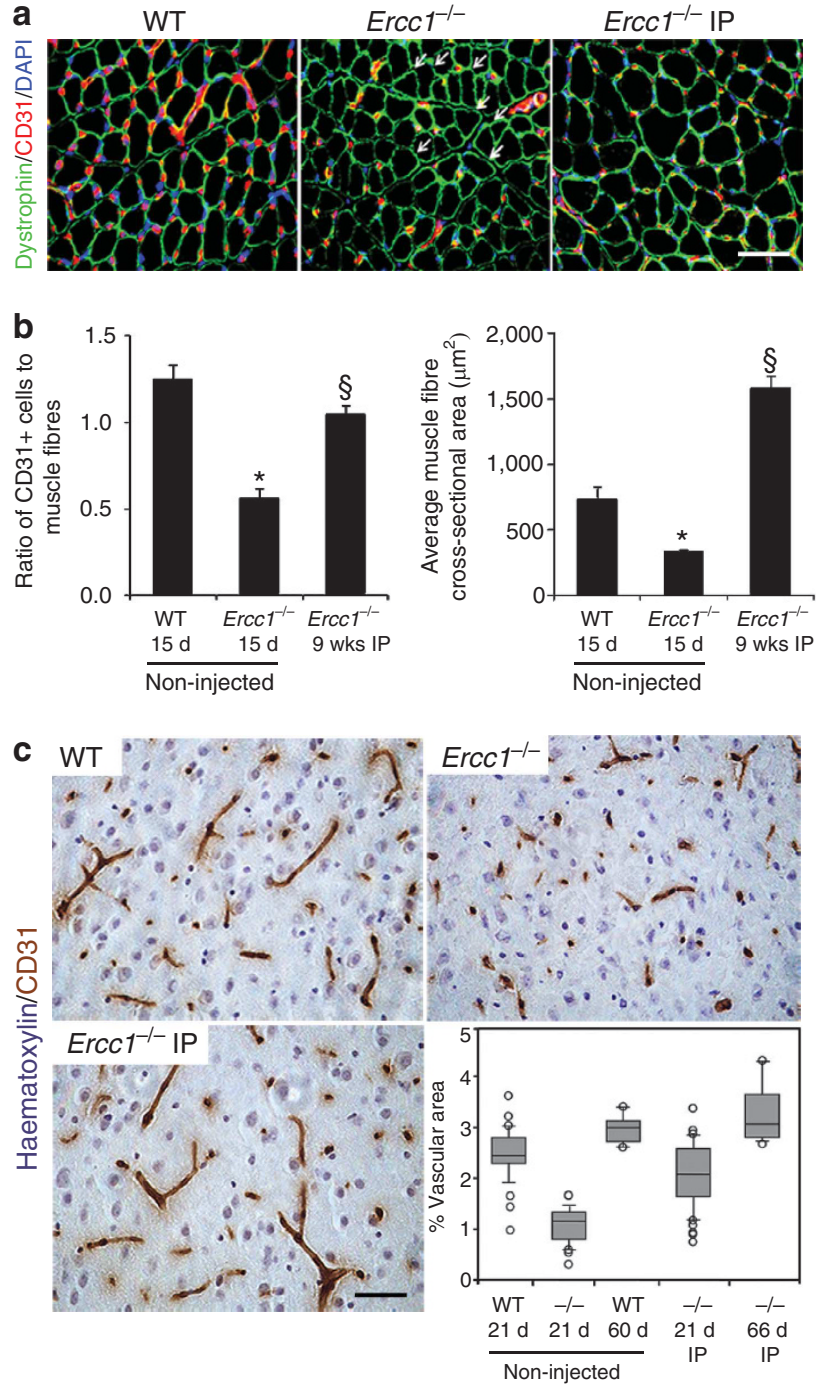

Figure 7 | Host muscle fibre size and tissue vascularization following intraperitoneal transplantation of young WT-MDSPCs. (a)

Representative sections from the gastrocnemius muscle of 15-day-old WT and $\mathrm{ErcC}^{-/-}$mice, as well as $\mathrm{ErcCl}^{-/-}$mice transplanted with young WT-MDSPCs at 17 days of age and allowed to live their full lifespan ( $\left.\mathrm{ErCl}^{-/-} \mathrm{IP}\right)$, immunostained for dystrophin (green), CD31 (red), and DAPI (blue). Arrows indicate myofibres lacking adjacent CD31+ cells (microvasculature). Scale bars $=100 \mu \mathrm{m}$. (b) Quantification of microvasculature and myofibre size in these mice. Images from 4-8 sections and 500-1,000 fibres from 3 animals per group were analysed. Reported are the ratio of CD31+ cells to dystrophin-positive muscle fibres (left) and the average muscle fibre size (cross-sectional area; right). Error bars indicate s.e.m. ${ }^{\star} P<0.001$, Student's t-test comparing the $\mathrm{Erccl}^{-/-}$ mice with WT mice and ${ }^{\S} P<0.05$, Tukey's test comparing untreated and treated $\mathrm{ErcCl}^{-/-}$mice; for myofibre size ${ }^{\star} P=0.002$, Mann-Whitney rank sum test comparing the $\mathrm{Ercc}^{-1}{ }^{-}$mice with WT mice and ${ }^{\S} P<0.05$, Dunn's Method comparing untreated and treated $\mathrm{Erccl}^{-/-}$mice. (c) Representative images from sections of the cerebral cortex of 21-dayold WT and $\mathrm{Ercc}^{-/-}$mice, and $\mathrm{Ercc}^{-/-}$mice transplanted with young WT-MDSPCs. Tissue sections were stained for CD31 to detect the microvasculature. The percent of the tissue area representing vasculature was quantified using bright field images and Northern Eclipse software. Each circle plotted represents an individual mouse. The horizontal bar is the median (50th percentile) area. The box represents the 25th-75th percentile, and the error bars represent the 10th-90th percentile. vations demonstrate that old, dysfunctional MDSPCs can be rescued by exogenous factors, rather than the cells being inherently defective. This is in contrast to aged haematopoietic stem cells, which appear to have an intrinsic defect (reviewed in ref. 57).

IP administration of young WT-MDSPCs to progeroid mice promoted growth of the animals, decreased skeletal muscle atrophy, and stimulated neovascularization in the brain and muscle, despite the fact that donor cells were not detected in these tissues (Figs 4 and 7). In addition, transplantation of young WT-MDSPCs directly into progeroid muscle led to muscle regeneration as well as host neovascularization, demonstrating a paracrine mechanism requiring secreted factors (Fig. 8). These observations suggest that the mechanism by which MDSPCs promote healthspan and lifespan extension is likely through the secretion of stimulatory factor(s) that promote regeneration and that cells from old hosts lose this secretory phenotype. This stimulatory factor may be necessary to overcome systemic ${ }^{48}$ or local ${ }^{58}$ negative regulators of tissue regeneration in aged hosts. Identification of these secreted factors and methods to rejuvenate adult stem cells ex vivo will be critical for regenerative medicine.

Our data demonstrate that transplanted MDSPCs can have systemic effects, leading to tissue regeneration in multiple organ systems. Therefore, MDSPCs may have therapeutic value for delaying ageing-related functional decline and treating human progerias. It will be of critical importance to test other adult stem cell types for a similar therapeutic effect.

\section{Methods}

Isolation of MDSPCs. All animal experiments were performed with the approval of the University of Pittsburgh Institutional Animal Care and Use Committee. The fraction of slow-adhering cells referred to as MDSPCs were isolated from the skeletal muscle of mice via a modified preplate technique ${ }^{21,22}$. Briefly, mouse skeletal muscle is enzymatically digested to a single-cell suspension and the cells are separated by their ability to adhere to collagen-coated flasks. The cells that adhere $2 \mathrm{~h}$ post-plating are defined as preplate 1 (pp1, Fig. 2). The non-adherent cells (pp2) are transferred to a fresh flask after $24 \mathrm{~h}$. This is repeated five times to isolate the slowly adhering, stem-cell-enriched MDSPC population (pp6 labelled ft). MDSPCs were isolated from young WT (14-21-day-old; f1 C57BL/6:FVB/n or C57BL/10J), adult WT (19-wk-old; f1), old WT (2-yr-old; f1), progeroid $\mathrm{Erccl}^{-1-}$ (14-21-dayold; f1), $\mathrm{Ercc1}^{-1 \Delta}$ (19-wk-old; f1) and DNA-repair-deficient $\mathrm{Xpa}^{-/-}$(21-day-old; C57BL/6) mice ( $n=3-4$ per strain), which were bred at the University of Pittsburgh Division of Laboratory Animal Research. MDSPCs were cultured in proliferation medium (PM): DMEM supplemented with $10 \%$ horse serum, $10 \%$ fetal bovine serum (FBS), $1 \%$ penicillin-streptomycin (all from Invitrogen), and $0.5 \%$ chick embryo extract (Accurate Chemical), on collagen-type I-coated flasks. Primary MEFs used for transplantation were prepared from E13-15 day-old embryos and cultured at $3 \% \mathrm{O}_{2}$ (ref. 32).

To isolate individual clones, $5 \times 10^{5}$ MDSPCs were resuspended in $500 \mu \mathrm{l}$ media and the FACS Aria cytometer (Becton-Dickinson) was used to place one MDSPC per well in a 96-well collagen-I-coated plate containing $100 \mu \mathrm{l}$ PM medium. Each well was manually checked to confirm the presence of a single cell. Cells were cultured at $37^{\circ} \mathrm{C}, 5 \% \mathrm{CO}_{2}$, in a humidified incubator and medium was changed every 2 days.

Proliferation in vitro. Cells were plated at a density of 2,000 cells per well in a collagen-type-I-coated 12-well plate. An imaging system (Automated Cell Technologies) equipped with a $\times 20$ objective was used to acquire images at 10 -min intervals over a period of $72 \mathrm{~h}$. Forty images at each time point were randomly selected and analysed from three independent experiments on three-to-four distinct cell lines. To estimate PDT, the experimental data sets for each population were curve-fit to the re-arranged Sherley model ${ }^{38,59}$, using nonlinear regression to determine the correlation coefficient and dividing fraction for each cell genotype.

The division time (DT) of individual cells within the population or of isolated clones was measured by determining the time between cytokinesis events using time-lapsed video recordings $(72 \mathrm{~h}$ in duration). At least 30 cells were tracked over time in at least 3 MDSPC populations per genotype. For clonal experiments, cells were analysed within 5 days after isolation of clones from pp6. Three-to-four clones were isolated from 2-3 MDSPC populations of each genotype. DT was, therefore, calculated from 6-12 clones per genotype (Supplementary Table S1).

Differentiation in vitro. To induce myogenic differentiation, MDSPCs were plated at high confluence in PM for 2-3 days then shifted to differentiation medium (DM: DMEM, 2\% FBS, 1\% penicillin-streptomycin). After 2-3 days, immunostaining 
a

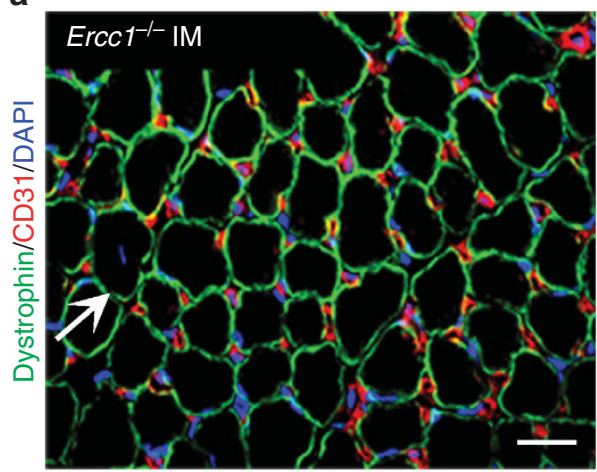

b

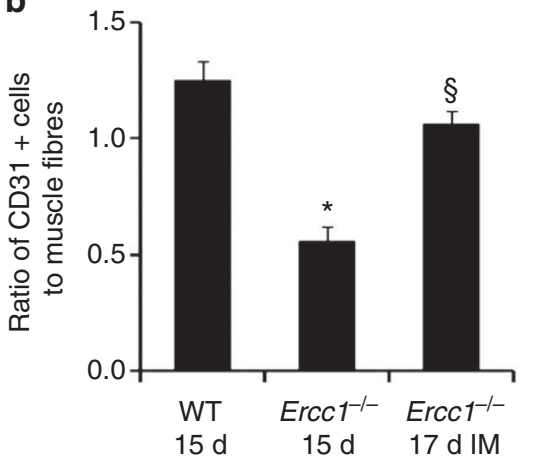

Non-injected

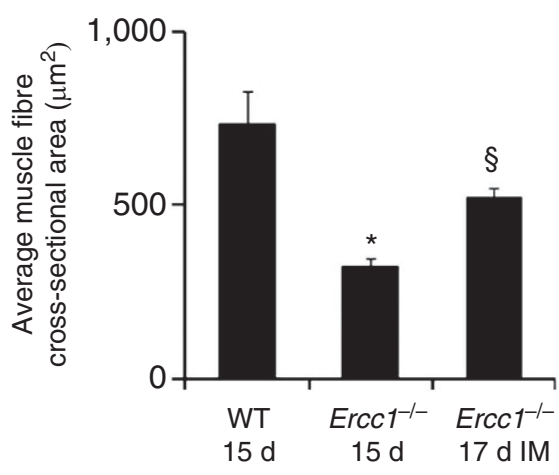

Non-injected c

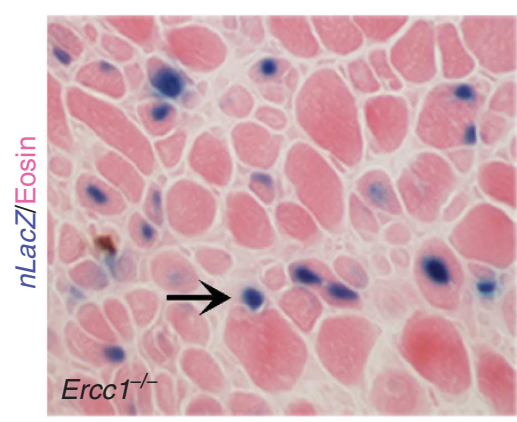

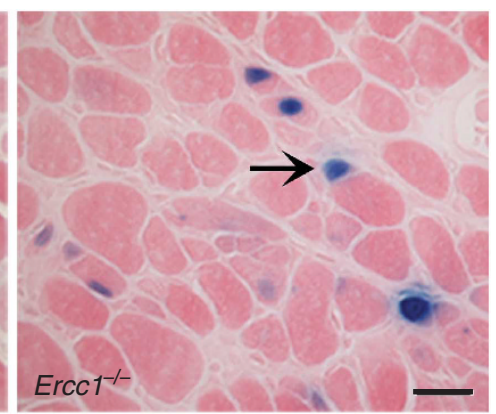

d

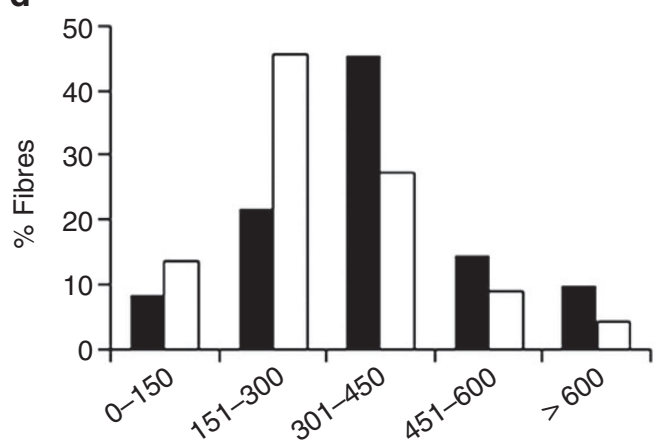

Fibre area $\left(\mu \mathrm{m}^{2}\right)$
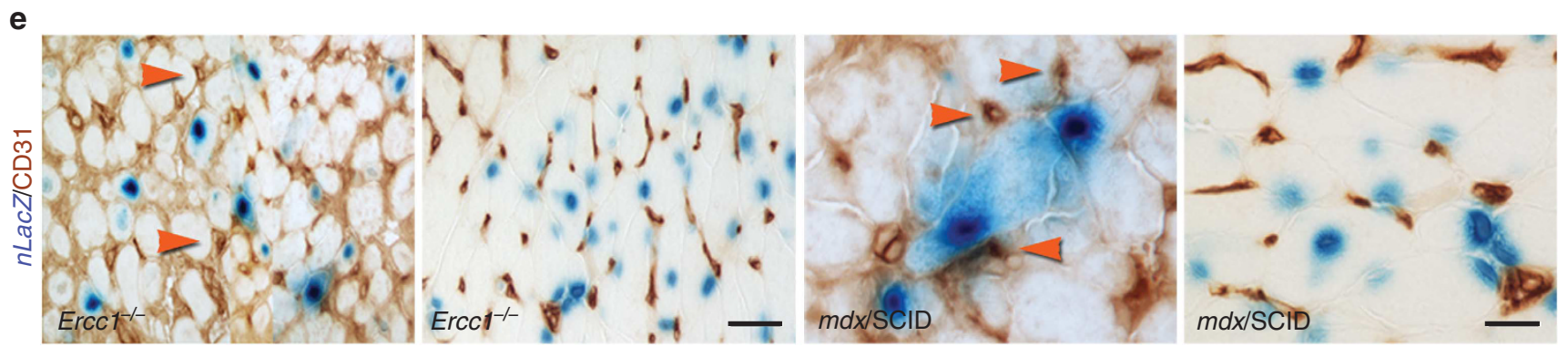

Figure 8 | Measurement of host muscle fibre size and tissue vascularization after intramuscular transplantation with young WT-MDSPCs. (a) The gastrocnemius muscle of 12-day-old $\operatorname{Ercc}^{-/-}$mice $(n=3$ mice) was injected with young WT-MDSPCs. Tissues were isolated 5 days later $(17 \mathrm{~d})$ and sections were immunostained for dystrophin (green), CD31 (red), and DAPI (blue) and compared with untreated 15-day-old WT and Ercc1 ${ }^{-/-}$mice (from Fig. 7). The white arrow denotes a centronucleated fibre. Scale bar $=100 \mu \mathrm{m}$. (b) Quantification of microvasculature and myofibre size in transplanted and untreated mice. Images from 4-8 sections from 3 animals per group were analysed. Reported is the ratio of CD31+ cells to dystrophin-positive muscle fibres (left), ${ }^{\S} P<0.05$ between untreated and treated $\mathrm{Ercc}^{-/-}$mice, Tukey's test. On the right is the average muscle fibre size (cross-sectional area), ${ }^{s_{P}} \mathrm{P}<0.05$ between untreated and treated $\mathrm{Ercc}^{-1-}$ mice, Dunn's test. Error bars indicate s.e.m. (c) Seventeen day-old progeroid Ercc $1^{-/-}$mice $(n=10)$ were injected IM with WT-MDSPCs expressing nuclear LacZ to determine whether donor cells integrate into muscle fibres and contribute to blood vessels. Five days later, the mice were euthanized and the gastrocnemius muscles were isolated for analysis. Muscle sections at the injection site were stained with X-gal, to detect donor cells, and eosin. Scale bar $=50 \mu \mathrm{m}$. (d) Histogram of the cross-sectional area distribution of myofibres in the treated gastrocnemius muscles (black bars) compared the contralateral non-injected muscle (white bars). Data were collected from 6,000 fibres from multiple sections of four mice per group. The distribution of fibre size is indicated on the $x$-axis, representing increasingly more mature fibres with increased size $(P<0.001$, MannWhitney rank sum test at 151-300 and 301-450). (e) Sections from the injected muscles stained for CD31 to identify blood vessels (dark brown) and X-gal to identify donor cells (blue). Red arrowheads indicate CD31+ capillary structures. Sections from dystrophic ( $m d x / S C I D)$ mice treated the same way are included as a positive control (right panels). Scale bars $=50 \mu \mathrm{m}$.

for f-MyHC, a marker of terminal myogenic differentiation, was performed. After cold methanol fixation, cells were blocked with $5 \%$ goat serum and incubated with f-MyHC mouse monoclonal antibody (1:250; Sigma-Aldrich), secondary biotinylated IgG (1:250; Vector Laboratories) for $1 \mathrm{~h}$, and streptavidin-594 (1:500; Sigma-Aldrich) for $15 \mathrm{~min}$ to fluorescently label the myotubes. To visualize the nuclei, the cultures were then incubated with DAPI $\left(4^{\prime}, 6^{\prime}\right.$ diamidino-2-phyenylindole, $100 \mathrm{n} \mathrm{ml}^{-1}$; Sigma-Aldrich) for $10 \mathrm{~min}$. For negative controls, the primary antibody was omitted or replaced with mouse or rabbit IgG. Fluorescent images were captured and processed using a Leica DMIRB microscope equipped with a
QImaging Retiga digital camera both using Northern Eclipse software (v. 6.0; Empix Imaging). We quantified the cells in at least 15 fields each from 3 replica platings of 4 independent experiments. Myogenic differentiation was calculated as the percent of cells (the number of nuclei, detected by DAPI stain) expressing $\mathrm{f}-\mathrm{MyHC}$.

For adipogenic, osteogenic, and chondrogenic differentiation, MDSPCs were seeded onto 6-well plates and lineage-specific differentiation media (Lonza) was added for 7 days. Expression of lineage-specific markers was measured by reverse transcriptase PCR (rtPCR) as described in Supplementary Methods and Supplementary Table S5. 
FACS. The preplate technique was utilized to obtain cells from skeletal muscle ${ }^{21}$. Red blood cells were eliminated using a lysis buffer before plating. After $24 \mathrm{~h}$, nonadherent cells in pp3 were collected, centrifuged, resuspended in mouse serum (1:10; Sigma-Aldrich) on ice and counted. Cells were then labelled with rat monoclonal antibodies against CD34, Sca-1, and CD45 conjugated to FITC, PE (both from BD Pharmingen), and APC, respectively (Invitrogen). A separate fraction of each cell pellet was stained with equivalent volumes of isotype control antibodies. Before analysis, 7-amino-actinomycin D (7-AAD) was added for exclusion of nonviable cells. Cells were analysed on a FACSAria cytometer using FACS Diva software, (Becton Dickinson). Sorted gates were defined based on separate cell portions that were labelled with equivalent amounts of appropriately conjugated isotype-matched control antibodies. Positive and negative gates were set by analysing isotype or unstained control samples in each analysis. The average percent of $\mathrm{Sca}-1^{+} / \mathrm{CD} 34^{+} / \mathrm{CD} 45^{-}$cells was normalized to pellet weight. To compare differentiation potential, an equal number of sorted cells from each group were plated in 48-well plates and differentiation was induced as described above.

Co-culture experiments. $\mathrm{Erccl}^{-1-}$ MDSPCs were plated in the lower compartment of Transwell Permeable Supports (Costar) in PM media at a density of 3,000 cells per well. WT-MDSPCs were seeded into $6.5 \mathrm{~mm}$ transwell membrane inserts at the same density in PM media and placed above the ERCC1-deficient MDSPCs. The 24-well plates containing the transwells were then placed in the LCI system for $72 \mathrm{~h}$ to acquire time-lapsed images to measure proliferation of the MDSPCs, as described above. As a control, each plate contained wells of $\mathrm{Ercc}^{-1-}$ MDSPCs without transwell membrane inserts. To measure the differentiation potential of $\mathrm{Erccl}^{-/-}$MDSPCs after co-culture, transwell inserts were removed after $72 \mathrm{~h}$ and the PM media was switched to DM. After 2-3 days, myogenic differentiation of the cells was tested by immunostaining for $\mathrm{f}-\mathrm{MyHC}$, as described above. To quantify differentiation, it was necessary to switch from transwell to conditioned media from young WT-MDSPCs to generate sufficient cells for quantitative immunodetection of myogenic differentiation markers. Young WTMDSPCs were cultured for 2 days in $25 \mathrm{~cm}^{2}$ collagen-coated flasks and then treated with differentiation media (DMEM medium supplemented with $2 \%$ FBS) for 3 days. The supernatant was collected and used as conditioned media. MDSPCs from 21-day-old $\mathrm{Erccl}^{-1-}$ mice and 2-yr-old WT mice were grown in the presence of this conditioned media or unconditioned differentiation media to determine the impact on myogenic differentiation.

Western blot. Three-to-four days after induction of myogenic differentiation, cells were lysed in Laemmli Sample Buffer (Bio-Rad), boiled for $5 \mathrm{~min}$, and centrifuged at 4,000 r.p.m. for $5 \mathrm{~min}$. Each sample was loaded on a 6\% SDS-polyacrylamide gel, which was run for $2 \mathrm{~h}$ by 100 volts and then transferred for $45 \mathrm{~min}$ by 20 volts in a semi-dry transferring apparatus. The membrane was blocked with $5 \%$ nonfat dry milk (Bio-rad) in Tris-buffered saline Tween-20 for $1 \mathrm{~h}$ and then incubated with mouse anti-MyHC (fast) monoclonal antibody (1:500, Sigma-Aldrich) and mouse anti- $\beta$-Actin monoclonal antibody (1:8,000, Sigma-Aldrich), respectively, overnight at $4^{\circ} \mathrm{C}$. After washing 3 times with Tris-buffered saline Tween-20, the membrane was incubated with rabbit anti-mouse IgG $(\mathrm{H}+\mathrm{L})(1: 5,000$, Pierce) for $1 \mathrm{~h}$ at room temperature. Blots were developed by ECL solution (Pierce). Differences in expression were measured by densitometry using software from SCION Corporation (provided by National Institutes of Health) and reported relative to $\beta$-Actin.

Tissue staining. Microvascular density was measured by co-staining for dystrophin to highlight muscle fibres and CD31 to identify endothelial cells present within microvessels ${ }^{35}$. Briefly, sections of frozen gastrocnemius muscle were formalin fixed, blocked with serum, and incubated with primary antibodies including CD31 (1:250; BD Biosciences) and dystrophin (1:300, Abcam) for $1 \mathrm{~h}$. Goat anti-rat IgG Alexa Fluor 594 (1:300, Invitrogen) and donkey anti-rabbit Alexa Fluor 488 (1:300, Invitrogen) were used to detect CD31 and dystrophin, respectively. DAPI was used to identify cell nuclei. For each image, the total number of muscle fibres and $\mathrm{CD} 31+$ cells were counted, and the ratio of CD31 + cells to muscle fibres was reported ${ }^{35}$. To detect $n L a c Z$ expression, tissue sections were fixed in $1 \%$ glutaraldehyde and incubated with 5-bromo-4-chloro-3-indolyl $\beta$-D-galactopyranoside (X-gal) solution for $3 \mathrm{~h}$ at $37^{\circ} \mathrm{C}$ and counterstained with haematoxylin and/or eosin. For details on co-localizing CD31 and $n L a c Z$ staining, see Supplementary Methods.

Statistics. Statistical analyses were carried out using SigmaStat (Jandel Scientific v2.0) software package. The paired Student's $t$-test or Mann-Whitney rank sum test was used for direct comparisons between treatment and control groups. For multiple comparisons, the one-way ANOVA or the Kruskal-Wallis one-way ANOVA on ranks was applied. Pairwise multiple comparisons were performed using the Tukey's Test or Dunn's Method following rank-based ANOVA. All values are expressed as the mean \pm s.d. (or s.e.m. where noted), and $P<0.05$ was considered significant.

\section{References}

1. Kirkwood, T. B. Understanding the odd science of aging. Cell 120, 437-447 (2005).
2. Sharpless, N. E. \& DePinho, R. A. How stem cells age and why this makes us grow old. Nat. Rev. Mol. Cell Biol. 8, 703-713 (2007).

3. Rossi, D. J., Jamieson, C. H. \& Weissman, I. L. Stems cells and the pathways to aging and cancer. Cell 132, 681-696 (2008).

4. Molofsky, A. V. et al. Increasing p16INK4a expression decreases forebrain progenitors and neurogenesis during ageing. Nature 443, 448-452 (2006).

5. Kuhn, H. G., Dickinson-Anson, H. \& Gage, F. H. Neurogenesis in the dentate gyrus of the adult rat: age-related decrease of neuronal progenitor proliferation. J. Neurosci. 16, 2027-2033 (1996).

6. Ryu, B. Y., Orwig, K. E., Oatley, J. M., Avarbock, M. R. \& Brinster, R. L. Effects of aging and niche microenvironment on spermatogonial stem cell selfrenewal. Stem Cells 24, 1505-1511 (2006).

7. Zhang, X., Ebata, K. T., Robaire, B. \& Nagano, M. C. Aging of male germ line stem cells in mice. Biol. Reprod. 74, 119-124 (2006).

8. Morrison, S. J., Wandycz, A. M., Akashi, K., Globerson, A. \& Weissman, I. L. The aging of hematopoietic stem cells. Nature Med. 2, 1011-1016 (1996).

9. Fuller, J. Hematopoietic stem cells and aging. Sci. Aging Knowledge Environ. 2002, pe11 (2002).

10. Conboy, I. M. \& Rando, T. A. The regulation of Notch signaling controls satellite cell activation and cell fate determination in postnatal myogenesis. Dev. Cell 3, 397-409 (2002).

11. Conboy, I. M., Conboy, M. J., Smythe, G. M. \& Rando, T. A. Notch-mediated restoration of regenerative potential to aged muscle. Science 302, 1575-1577 (2003)

12. Shefer, G., Van de Mark, D. P., Richardson, J. B. \& Yablonka-Reuveni, Z. Satellite-cell pool size does matter: defining the myogenic potency of aging skeletal muscle. Dev. Biol. 294, 50-66 (2006).

13. Collins, C. A., Zammit, P. S., Ruiz, A. P., Morgan, J. E. \& Partridge, T. A. A population of myogenic stem cells that survives skeletal muscle aging. Stem Cells 25, 885-894 (2007).

14. Sethe, S., Scutt, A. \& Stolzing, A. Aging of mesenchymal stem cells. Ageing Res. Rev. 5, 91-116 (2006).

15. Stolzing, A., Jones, E., McGonagle, D. \& Scutt, A. Age-related changes in human bone marrow-derived mesenchymal stem cells: consequences for cell therapies. Mech. Ageing Dev. 129, 163-173 (2008).

16. Scaffidi, P. \& Misteli, T. Lamin A-dependent misregulation of adult stem cells associated with accelerated ageing. Nat. Cell Biol. 10, 452-459 (2008).

17. Huang, S. C. et al. Mechanical strain modulates age-related changes in the proliferation and differentiation of mouse adipose-derived stromal cells. BMC Cell Biol. 11, 18 (2010).

18. Grounds, M. D. Age-associated changes in the response of skeletal muscle cells to exercise and regeneration. Ann. N. Y. Acad. Sci. 854, 78-91 (1998).

19. Welle, S. Cellular and molecular basis of age-related sarcopenia. Can. J. Appl. Physiol. 27, 19-41 (2002).

20. Thompson, L. V. Age-related muscle dysfunction. Exp. Gerontol. 44, 106-111 (2009).

21. Qu-Petersen, Z. et al. Identification of a novel population of muscle stem cells in mice: potential for muscle regeneration. J. Cell Biol. 157, 851-864 (2002).

22. Gharaibeh, B. et al. Isolation of a slowly adhering cell fraction containing stem cells from murine skeletal muscle by the preplate technique. Nat. Protoc. 3 , 1501-1509 (2008).

23. Peng, H. et al. Synergistic enhancement of bone formation and healing by stem cell-expressed VEGF and bone morphogenetic protein-4. J. Clin. Invest. 110, 751-759 (2002).

24. Payne, T. R. et al. A relationship between vascular endothelial growth factor, angiogenesis, and cardiac repair after muscle stem cell transplantation into ischemic hearts. J. Am. Coll. Cardiol. 50, 1677-1684 (2007).

25. Oshima, H. et al. Differential myocardial infarct repair with muscle stem cells compared to myoblasts. Mol. Ther. 12, 1130-1141 (2005).

26. Crisan, M. et al. A perivascular origin for mesenchymal stem cells in multiple human organs. Cell Stem Cell 3, 301-313 (2008).

27. Shen, J. et al. Transplantation of mesenchymal stem cells from young donors delays aging in mice. Sci. Rep. 1, 67 doi:10.1038/srep00067 (2011).

28. Sun, Y. et al. Rescuing replication and osteogenesis of aged mesenchymal stem cells by exposure to a young extracellular matrix. FASEB J. 25, 1474-1485 (2011)

29. Niedernhofer, L. J. et al. A new progeroid syndrome reveals that genotoxic stress suppresses the somatotroph axis. Nature 444, 1038-1043 (2006).

30. Gregg, S. Q., Robinson, A. R. \& Niedernhofer, L. J. Physiological consequences of defects in ERCC1-XPF DNA repair endonuclease. DNA Repair (Amst.) 10, 781-791 (2011).

31. Weeda, G. et al. Disruption of mouse ERCC1 results in a novel repair syndrome with growth failure, nuclear abnormalities and senescence. Curr. Biol. 7, 427-439 (1997).

32. Dolle, M. E. et al. Increased genomic instability is not a prerequisite for shortened lifespan in DNA repair deficient mice. Mutat. Res. 596, 22-35 (2006).

33. Chipchase, M. D., O’Neill, M. \& Melton, D. W. Characterization of premature liver polyploidy in DNA repair (Ercc1)-deficient mice. Hepatology 38, 958-966 (2003). 
34. Vo, N. et al. Accelerated aging of intervertebral discs in a mouse model of progeria. J. Orthop. Res. 28, 1600-1607 (2010).

35. Deasy, B. M. et al. Effect of VEGF on the regenerative capacity of muscle stem cells in dystrophic skeletal muscle. Mol. Ther. 17, 1788-1798 (2009).

36. Jankowski, R. J., Deasy, B. M. \& Huard, J. Muscle-derived stem cells. Gene Ther 9, 642-647 (2002).

37. de Vries, A. et al. Increased susceptibility to ultraviolet-B and carcinogens of mice lacking the DNA excision repair gene XPA. Nature 377, 169-173 (1995).

38. Deasy, B. M. et al. Modeling stem cell population growth: incorporating terms for proliferative heterogeneity. Stem Cells 21, 536-545 (2003).

39. Hoffman, E. P., Monaco, A. P., Feener, C. C. \& Kunkel, L. M. Conservation of the Duchenne muscular dystrophy gene in mice and humans. Science 238, 347-350 (1987).

40. Mitchell, P. O. et al. Sca-1 negatively regulates proliferation and differentiation of muscle cells. Dev. Biol. 283, 240-252 (2005).

41. Joe, A. W. et al. Muscle injury activates resident fibro/adipogenic progenitors that facilitate myogenesis. Nat. Cell Biol. 12, 153-163 (2010).

42. Hidestrand, M. et al. Sca-1-expressing nonmyogenic cells contribute to fibrosis in aged skeletal muscle. J. Gerontol. A Biol. Sci. Med. Sci. 63, 566-579 (2008).

43. d'Albis, A., Couteaux, R., Janmot, C., Roulet, A. \& Mira, J. C. Regeneration after cardiotoxin injury of innervated and denervated slow and fast muscles of mammals. Myosin isoform analysis. Eur. J. Biochem. 174, 103-110 (1988).

44. Peng, H. et al. VEGF improves, whereas sFlt1 inhibits, BMP2-induced bone formation and bone healing through modulation of angiogenesis. J. Bone Miner. Res. 20, 2017-2027 (2005).

45. Payne, T. R. et al. Regeneration of dystrophin-expressing myocytes in the $\mathrm{mdx}$ heart by skeletal muscle stem cells. Gene Ther. 12, 1264-1274 (2005).

46. Janzen, V. et al. Stem-cell ageing modified by the cyclin-dependent kinase inhibitor p16INK4a. Nature 443, 421-426 (2006).

47. Conboy, I. M. et al. Rejuvenation of aged progenitor cells by exposure to a young systemic environment. Nature 433, 760-764 (2005).

48. Brack, A. S. et al. Increased Wnt signaling during aging alters muscle stem cell fate and increases fibrosis. Science 317, 807-810 (2007).

49. Zhang, J. et al. A human iPSC model of Hutchinson Gilford Progeria reveals vascular smooth muscle and mesenchymal stem cell defects. Cell Stem Cell 8 31-45 (2011).

50. Baxter, M. A. et al. Study of telomere length reveals rapid aging of human marrow stromal cells following in vitro expansion. Stem Cells 22, 675-682 (2004).

51. Gopinath, S. D. \& Rando, T. A. Stem cell review series: aging of the skeletal muscle stem cell niche. Aging Cell 7, 590-598 (2008).

52. van der Lugt, N. M. et al. Posterior transformation, neurological abnormalities, and severe hematopoietic defects in mice with a targeted deletion of the bmi-1 proto-oncogene. Genes Dev. 8, 757-769 (1994).

53. Park, I. K. et al. Bmi-1 is required for maintenance of adult self-renewing haematopoietic stem cells. Nature 423, 302-305 (2003).

54. Nishino, J., Kim, I., Chada, K. \& Morrison, S. J. Hmga2 promotes neural stem cell self-renewal in young but not old mice by reducing p16Ink4a and p19Arf expression. Cell 135, 227-239 (2008).

55. Nijnik, A. et al. DNA repair is limiting for haematopoietic stem cells during ageing. Nature 447, 686-690 (2007).
56. Rossi, D. J. et al. Deficiencies in DNA damage repair limit the function of haematopoietic stem cells with age. Nature 447, 725-729 (2007).

57. Brunet, A. \& Rando, T. A. Ageing: from stem to stern. Nature 449, 288-291 (2007).

58. Carlson, M. E., Hsu, M. \& Conboy, I. M. Imbalance between pSmad3 and Notch induces CDK inhibitors in old muscle stem cells. Nature 454, 528-532 (2008).

59. Sherley, J. L., Stadler, P. B. \& Stadler, J. S. A quantitative method for the analysis of mammalian cell proliferation in culture in terms of dividing and nondividing cells. Cell Prolif. 28, 137-144 (1995).

\section{Acknowledgements}

We thank Ayse Baybars for technical assistance, Minakshi Poddar for performing western blot experiments, Vaishali Patil for maintaining the mouse colony, Alison Logar for assistance with flow cytometry and Dr. Bridget Deasy (University of Pittsburgh, Pittsburgh, PA) for sharing her expertise, methodology and established protocols in regards to the live-cell imaging (LCI) system. This work was supported in part by The Ellison Medical Foundation AG-NS-0303-05 and ES016114, AG033907-01A1, and AR051456 from the National Institutes of Health, the Henry J. Mankin Endowed Chair at the University of Pittsburgh, William F. and Jean W. Donaldson endowed chair at the Children's Hospital of Pittsburgh. J.S.T. was supported by NIH post-doctoral fellowship F30 AG032816. The experiments were approved by the Institutional Animal Care and Use Committee of the University of Pittsburgh

\section{Author contributions}

M.L. contributed to all experiments, analysed the data, did statistical analysis, prepared the figures and wrote the manuscript. A.R.R. developed the animal cohorts and assisted with the in vivo experiments. A.L. isolated the stem cell lines and contributed to the in vitro experiments. M.S. performed the rtPCR. J.S.T. and C.H.F. contributed to the in vivo experiments and healthspan data analysis. J.M.F. contributed to the in vitro experiments and angiogenesis studies. B.A. did all of the tissue staining, imaging and quantification. J.H. supervised in vitro experiments. L.J.N. supervised in vivo work. M.L., P.D.R, L.J.N., and J.H. contributed to data analysis and manuscript preparation.

\section{Additional information}

Supplementary Information accompanies this paper at http://www.nature.com/ naturecommunications

Competing financial interests: The authors, except J.H., declare no competing financial interests. J.H. receives consulting fees from Cook MyoSite.

Reprints and permission information is available online at http://npg.nature.com/ reprintsandpermissions/

How to cite this article: Lavasani, M. et al. Muscle-derived stem/progenitor cell dysfunction limits healthspan and lifespan in a murine progeria model. Nat. Commun. 3:608 doi: 10.1038 /ncomms1611 (2012).

License: This work is licensed under a Creative Commons Attribution-NonCommercialShare Alike 3.0 Unported License. To view a copy of this license, visit http:// creativecommons.org/licenses/by-nc-sa/3.0/ 